\title{
On the existence of Feller semigroups with discontinuous coefficients II
}

\author{
Kazuaki Taira* \\ Institute of Mathematics, University of Tsukuba, Tsukuba 305-8571, Japan \\ E-mail: taira@math.tsukuba.ac.jp
}

Dedicated to Professor Hikosaburo Komatsu on the occasion of his 70th birthday

\begin{abstract}
This paper is devoted to the functional analytic approach to the problem of existence of Markov processes with Dirichlet boundary condition, oblique derivative boundary condition and first-order Ventcel' boundary condition for second-order, uniformly elliptic differential operators with discontinuous coefficients. More precisely, we construct Feller semigroups associated with absorption, reflection, drift and sticking phenomena at the boundary. The approach here is distinguished by the extensive use of the ideas and techniques characteristic of the recent developments in the Calderón-Zygmund theory of singular integral operators with non-smooth kernels.
\end{abstract}

\section{Introduction and Main Results}

Let $\Omega$ be a bounded domain in Euclidean space $\mathbf{R}^{N}, N \geq 3$, with boundary $\partial \Omega$ of class $C^{1,1}$. We consider a second-order, elliptic differential operator $A$ with real discontinuous coefficients of the form

$$
A u:=\sum_{i, j=1}^{N} a^{i j}(x) \frac{\partial^{2} u}{\partial x_{i} \partial x_{j}}+\sum_{i=1}^{N} b^{i}(x) \frac{\partial u}{\partial x_{i}}+c(x) u .
$$

In the case of continuous coefficients $a^{i j}(x)$, an $L^{p}$ Schauder theory has been elaborated for second-order, uniformly elliptic differential operators (see [1]). However, the situation becomes rather difficult if we try to allow discontinuity on the $a^{i j}(x)$. In fact, it is known (see [2], [3], [4]) that arbitrary discontinuity of the $a^{i j}(x)$ breaks down as the $L^{p}$ Schauder theory, except for the two-dimensional case $(N=2)$. In order to handle with the multidimensional case $(N \geq 3)$, additional conditions on the $a^{i j}(x)$ should be required. Here we shall see that the relevant condition is that the coefficients $a^{i j}(x)$ belong to the Sarason class VMO

\footnotetext{
${ }^{*}$ Supported in part by Grant-in-Aid for General Scientific Research (No. 16340031), Ministry of Education, Culture, Sports, Science and Technology, Japan.

2000 Mathematics Subject Classification. Primary 47D07, 35J25; Secondary 47D05, $60 \mathrm{~J} 35,60 \mathrm{~J} 60$

Keyword. Singular integral, Feller semigroup, elliptic operator with VMO coefficients, Ventcel' boundary condition
} 
of functions with vanishing mean oscillation. We remark that VMO consists of the John-Nirenberg class BMO of functions with bounded mean oscillation whose integral oscillation over balls shrinking to a point converge uniformly to zero (see Section 2 for the precise definitions and references).

Our approach here is distinguished by the extensive use of the CalderónZygmund theory of singular integrals (see [5]). Since second-order elliptic differential operators are pseudo-differential operators only if the coefficients are smooth, we can not make use of the theory of pseudo-differential operators as in the previous work [6]. It should be emphasized that singular integral operators provide a powerful tool to deal with smoothness of solutions of partial differential equations, with minimal assumptions of regularity on the coefficients. Several recent developments in the theory of singular integrals (see [7], [8], [9], [10]) have made possible further progress in the study of elliptic boundary value problems with discontinuous coefficients and hence in the study of Markov processes. The presentation of these new results is the main purpose of this paper.

Throughout this paper, we assume that the coefficients $a^{i j}(x), b^{i}(x)$ and $c(x)$ of the differential operator $A$ satisfy the following three conditions (1), (2) and (3):

(1) $a^{i j}(x) \in \mathrm{VMO} \cap L^{\infty}(\Omega), a^{i j}(x)=a^{j i}(x)$ for almost all $x \in \Omega$ and there exist a constant $\lambda>0$ such that

$$
\frac{1}{\lambda}|\xi|^{2} \leq \sum_{i, j=1}^{N} a^{i j}(x) \xi_{i} \xi_{j} \leq \lambda|\xi|^{2} \quad \text { for almost all } x \in \Omega \text { and all } \xi \in \mathbf{R}^{N} \text {. }
$$

(2) $b^{i}(x) \in L^{\infty}(\Omega)$.

(3) $c(x) \in L^{\infty}(\Omega)$ and $c(x) \leq 0$ for almost all $x \in \Omega$.

The differential operator $A$ is called a diffusion operator which describes analytically a strong Markov process with continuous paths in the interior $\Omega$ such as Brownian motion.

Moreover, we consider a first-order, boundary operator of the form

$$
L u:=\mu\left(x^{\prime}\right) \frac{\partial u}{\partial \mathbf{n}}+\beta\left(x^{\prime}\right) \cdot u+\gamma\left(x^{\prime}\right) u-\delta\left(x^{\prime}\right)\left(\left.A u\right|_{\partial \Omega}\right) \quad \text { on } \partial \Omega .
$$

We assume that the coefficients $\mu\left(x^{\prime}\right), \beta\left(x^{\prime}\right), \gamma\left(x^{\prime}\right)$ and $\delta\left(x^{\prime}\right)$ of the boundary operator $L$ satisfy the following four conditions (4), (5), (6) and (7):

(4) $\mu\left(x^{\prime}\right)$ is a Lipschitz continuous function on $\partial \Omega$ and $\mu\left(x^{\prime}\right) \geq 0$ on $\partial \Omega$.

(5) $\beta\left(x^{\prime}\right)$ is a Lipschitz continuous vector field on $\partial \Omega$.

(6) $\gamma\left(x^{\prime}\right)$ is a Lipschitz continuous function on $\partial \Omega$ and $\gamma\left(x^{\prime}\right) \leq 0$ on $\partial \Omega$.

(7) $\delta\left(x^{\prime}\right)$ is a Lipschitz continuous function on $\partial \Omega$ and $\delta\left(x^{\prime}\right) \geq 0$ on $\partial \Omega$.

(8) $\mathbf{n}=\left(n_{1}, n_{2}, \ldots, n_{N}\right)$ is the unit interior normal to the boundary $\partial \Omega$.

The boundary condition $L$ is called a first-order Ventcel' boundary condition (see [11]). The four terms of $L$

$$
\mu\left(x^{\prime}\right) \frac{\partial u}{\partial \mathbf{n}}, \quad \beta\left(x^{\prime}\right) \cdot u, \quad \gamma\left(x^{\prime}\right) u, \quad \delta\left(x^{\prime}\right)\left(\left.A u\right|_{\partial \Omega}\right)
$$


are supposed to correspond to the reflection phenomenon, the drift phenomenon along the boundary, the absorption phenomenon and the sticking (or viscosity) phenomenon, respectively.

Let $C(\bar{\Omega})$ be the Banach space of real-valued, continuous functions on the closure $\bar{\Omega}=\Omega \cup \partial \Omega$, equipped with the maximum norm

$$
\|f\|_{C(\bar{\Omega})}=\max _{x \in \bar{\Omega}}|f(x)|, \quad f \in C(\bar{\Omega}) .
$$

A strongly continuous semigroup $\left\{T_{t}\right\}_{t \geq 0}$ on the space $C(\bar{\Omega})$ is called a Feller semigroup if it is non-negative and contractive on $C(\bar{\Omega})$, that is,

$$
f \in C(\bar{\Omega}), 0 \leq f(x) \leq 1 \quad \text { on } \bar{\Omega} \Longrightarrow 0 \leq T_{t} f(x) \leq 1 \quad \text { on } \bar{\Omega} .
$$

It is known (see [12], [13]) that if $T_{t}$ is a Feller semigroup on $C(\bar{\Omega})$, then there exists a unique Markov transition function $p_{t}(x, \cdot)$ on $\bar{\Omega}$ such that

$$
T_{t} f(x)=\int_{\bar{\Omega}} p_{t}(x, d y) f(y), \quad f \in C(\bar{\Omega}) .
$$

Furthermore, it can be shown (see [14]) that the function $p_{t}(x, \cdot)$ is the transition function of some strong Markov process whose paths are right-continuous and have no discontinuities other than jumps; hence the value $p_{t}(x, E)$ expresses the transition probability that a Markovian particle starting at position $x$ will be found in the set $E$ at time $t$.

The purpose of this paper is devoted to the functional analytic approach to the problem of existence of Markov processes in probability theory. More precisely, we consider the following problem:

Problem. Conversely, given analytic data $(A, L)$, can we construct a Feller semigroup $\left\{T_{t}\right\}_{t \geq 0}$ whose infinitesimal generator $\mathfrak{A}$ is characterized by $(A, L)$ ?

In the previous paper [15], we have constructed Feller semigroups with Dirichlet conditions for second-order uniformly elliptic integro-differential operators with discontinuous coefficients (see [15, Theorem 1.2]). Rephrased, we have proved that there exists a Feller semigroup corresponding to such a diffusion phenomenon that a Markovian particle moves both by jumps and continuously in the state space until it dies at the time when it reaches the boundary.

The next theorem asserts that there exists a Feller semigroup corresponding to such a diffusion phenomenon that a Markovian particle moves continuously in the state space, with absorption, reflection, drift and sticking phenomena at the boundary:

Theorem 1.1. If $N<p<\infty$, we define a linear operator $\mathfrak{A}$ from $C(\bar{\Omega})$ into itself as follows:

(a) The domain $D(\mathfrak{A})$ is the set

$$
D(\mathfrak{A})=\left\{u \in W^{2, p}(\Omega): A u \in C(\bar{\Omega}), L u=0 \text { on } \partial \Omega\right\} .
$$

(b) $\mathfrak{A} u=A u, u \in D(\mathfrak{A})$. 
Here $A u$ and Lu are taken in the sense of distributions.

Assume that the functions $\mu\left(x^{\prime}\right)$ and $\gamma\left(x^{\prime}\right)$ satisfy the following two conditions (H.1) and (H.2):

$$
\mu\left(x^{\prime}\right)>0 \quad \text { on } \partial \Omega
$$

and

$$
\gamma\left(x^{\prime}\right)<0 \quad \text { on } \partial \Omega \text {. }
$$

Then the operator $\mathfrak{A}$ is the infinitesimal generator of a Feller semigroup on $C(\bar{\Omega})$.

Remark 1.1. The domain $D(\mathfrak{A})$ does not depend on $p$, for $N<p<\infty$ (see Subsection 6.4).

The crucial point in the proof of Theorem 1.1 is that we consider the term $\delta\left(x^{\prime}\right)\left(\left.A u\right|_{\partial \Omega}\right)$ of sticking phenomenon in the boundary condition

$$
L u=\mu\left(x^{\prime}\right) \frac{\partial u}{\partial \mathbf{n}}+\beta\left(x^{\prime}\right) \cdot u+\gamma\left(x^{\prime}\right) u-\delta\left(x^{\prime}\right)\left(\left.A u\right|_{\partial \Omega}\right) \quad \text { on } \partial \Omega
$$

as a term of perturbation of the oblique derivative boundary condition $\left(\delta\left(x^{\prime}\right) \equiv\right.$ 0)

$$
L_{0} u:=\mu\left(x^{\prime}\right) \frac{\partial u}{\partial \mathbf{n}}+\beta\left(x^{\prime}\right) \cdot u+\gamma\left(x^{\prime}\right) u \quad \text { on } \partial \Omega .
$$

To do this, in Section 5 we prove the following generation theorem for Feller semigroups with oblique derivative boundary condition:

Theorem 1.2. If $N<p<\infty$, we define a linear operator $\mathfrak{A}_{N}$ from $C(\bar{\Omega})$ into itself as follows:

(a) The domain $D\left(\mathfrak{A}_{N}\right)$ is the set

$$
D\left(\mathfrak{A}_{N}\right)=\left\{u \in W^{2, p}(\Omega): A u \in C(\bar{\Omega}), L_{0} u=0 \text { on } \partial \Omega\right\},
$$

where

$$
L_{0} u:=\mu\left(x^{\prime}\right) \frac{\partial u}{\partial \mathbf{n}}+\beta\left(x^{\prime}\right) \cdot u+\gamma\left(x^{\prime}\right) u \quad \text { on } \partial \Omega .
$$

(b) $\mathfrak{A}_{N} u=A u, u \in D\left(\mathfrak{A}_{N}\right)$.

Here $A u$ and $L_{0} u$ are taken in the sense of distributions.

Assume that the functions $\mu\left(x^{\prime}\right)$ and $\gamma\left(x^{\prime}\right)$ satisfy the conditions (H.1) and (H.2). Then the operator $\mathfrak{A}_{N}$ is the infinitesimal generator of a Feller semigroup on $C(\bar{\Omega})$.

Remark 1.2. The domain $D\left(\mathfrak{A}_{N}\right)$ does not depend on $p$, for $N<p<\infty$ (see Subsection 5.4).

Rephrased, Theorem 1.2 asserts that there exists a Feller semigroup corresponding to such a diffusion phenomenon that a Markovian particle moves continuously in the state space, with absorption, reflection and drift phenomena at the boundary. 
The rest of this paper is organized as follows. In Section 2 we recall some basic definitions and results concerning BMO and VMO functions from real analysis. Section 3 provides a brief description of the basic definitions and results about Feller semigroups associated with Markov processes in probability theory, which forms a functional analytic background for the proof of Theorems 1.1 and 1.2. In particular, we formulate a version of the Hille-Yosida theorem adapted to the present context (Theorem 3.1). Moreover, we give two useful criteria in order that a linear operator be the infinitesimal generator of some Feller semigroup (Theorem 3.2 and Corollary 3.3). In Section 4 we consider the Dirichlet problem for the diffusion operator with VMO coefficients in the framework of Sobolev spaces of $L^{p}$ style, and prove an existence and uniqueness theorem for the Dirichlet problem (Theorem 4.1). The uniqueness result in Theorem 4.1 follows from a variant of the Bakel'man-Aleksandrov maximum principle in the framework of Sobolev spaces, essentially due to Bony [16] (Theorem 4.4). In Section 5 we study the oblique derivative problem in the framework of Sobolev spaces of $L^{p}$ style, and prove an existence and uniqueness theorem for the oblique derivative problem with VMO coefficients (Theorem 5.1). The uniqueness result in Theorem 5.1 follows from a variant of the Bakel'man-Aleksandrov maximum principle in the framework of Sobolev spaces due to Lieberman [17] (Theorem 5.3). Subsection 5.3 is devoted to the proof of Theorem 1.2. The purpose of Section 6 is to prove a general existence theorem for Feller semigroups with Ventcel' boundary condition in terms of elliptic boundary value problems (Theorem 6.9), following the main idea of Taira [13]. Intuitively, Theorem 6.9 asserts that we can "piece together" a Markov process on the boundary $\partial \Omega$ with $A$-diffusion in the interior $\Omega$ to construct a Markov process on the closure $\bar{\Omega}=\Omega \cup \partial \Omega$. The final Section 7 is devoted to the proof of Theorem 1.1. More precisely, we make use of a generation theorem for Feller semigroups with oblique derivative boundary condition $L_{0}$ to verify all the conditions of a version of the Hille-Yosida theorem (Theorem 3.2) for the operator $\mathfrak{A}$ defined by formula (1.3). In the appendix we formulate various maximum principles for second-order elliptic differential operators with discontinuous coefficients such as the weak and strong maximum principles (Theorems A.1 and A.3) and the boundary point lemma (Lemma A.2) in the framework of Sobolev spaces of $L^{p}$ style.

\section{BMO and VMO Functions}

In this section we recall some basic definitions and results concerning BMO and VMO functions on $\mathbf{R}^{N}$ from real analysis. For more thorough treatments of this subject, the reader might be referred to Garnett [18] and Torchinsky [19].

First we let

$$
\begin{aligned}
L_{\text {loc }}^{1}\left(\mathbf{R}^{N}\right)= & \text { the space of equivalence classes of Lebesgue measurable } \\
& \text { functions on } \mathbf{R}^{N} \text { which are integrable on every compact } \\
& \text { subset of } \mathbf{R}^{N} .
\end{aligned}
$$

The elements of $L_{\text {loc }}^{1}\left(\mathbf{R}^{N}\right)$ are called locally integrable functions on $\mathbf{R}^{N}$. A function $f \in L_{\mathrm{loc}}^{1}\left(\mathbf{R}^{N}\right)$ is said to be of bounded mean oscillation, $f \in \mathrm{BMO}$, 
if it satisfies the condition (see [20])

$$
\|f\|_{*}:=\sup _{B} \frac{1}{|B|} \int_{B}\left|f(x)-f_{B}\right| d x<\infty,
$$

where the supremum is taken over all balls $B$ in $\mathbf{R}^{N}$ and $f_{B}$ is the average of $f$ over $B$

$$
f_{B}:=\frac{1}{|B|} \int_{B} f(x) d x .
$$

The quantity $\|\cdot\|_{*}$ is called the $B M O$ norm. This is not properly a norm, since any function which is constant almost everywhere has zero oscillation. However, it is easy to see that these are the only functions having zero oscillation. Therefore, we view the class BMO as the quotient space of the above space by the space of constant functions. In other words, two functions which differ by a constant coincide as functions in the class BMO. Then it should be emphasized (see [21]) that the quantity $\|f\|_{*}$ defines a norm on the quotient space BMO/R.

Next we introduce a subspace of BMO functions whose BMO norm over a ball vanishes as the radius of the ball tends to zero. More precisely, if $f \in \mathrm{BMO}$ and $r>0$, then we let

$$
\eta(r):=\sup _{\rho \leq r} \frac{1}{|B|} \int_{B}\left|f(x)-f_{B}\right| d x,
$$

where the supremum is taken over all balls $B$ with radius $\rho \leq r$. A function $f \in \mathrm{BMO}$ has vanishing mean oscillation, $f \in \mathrm{VMO}$, if it satisfies the condition (see $[22]$ )

$$
\lim _{r \downarrow 0} \eta(r)=0 .
$$

The function $\eta(r)$ will be referred as the VMO modulus of $f$. The assumption that $f \in \mathrm{VMO}$ means a kind of continuity in the average sense, not in the pointwise sense.

It is easy to verify the following two assertions (i) and (ii):

(i) Uniformly continuous functions which belong to BMO are VMO functions.

(ii) VMO is a closed subspace of BMO.

The next theorem collects some important results concerning VMO functions (see [18, Chapter VI, Theorem 5.1], [19, Chapter VIII]):

Theorem 2.1. For a function $f \in \mathrm{BMO}$, the following three conditions (i), (ii) and (iii) are equivalent:

(i) $f$ is in VMO.

(ii) $f$ is in the BMO closure of uniformly continuous functions that belong to $B M O$.

(iii) $\lim _{y \rightarrow 0}\|f(\cdot-y)-f(\cdot)\|_{*}=0$, where $f(x-y)$ is the translation of $f(x)$ by $y$-units.

Example 2.1. (i) $\ln |x| \in \mathrm{BMO}$, but $\ln |x| \notin \mathrm{VMO}$. 
(ii) $\ln |\ln | x|| \in$ VMO.

Remark 2.1. Let $\Omega$ be a bounded domain of $\mathbf{R}^{n}, n \geq 3$. Then it should be emphasized that, by replacing the ball $B$ above by the intersection $B \cap \Omega$ we obtain the definitions of $\operatorname{BMO}(\Omega)$ and $\operatorname{VMO}(\Omega)$. Given a function defined on $\Omega$ that belongs to $\operatorname{BMO}(\Omega)$ (resp. $\operatorname{VMO}(\Omega)$ ), we can extend it to the whole $\mathbf{R}^{N}$ preserving its BMO (resp. VMO) character (see [23, Proposition 1.3]).

\section{Feller Semigroups and Markov Processes}

This section provides a brief description of the basic definitions and results about a class of semigroups, Feller semigroups, associated with Markov processes in probability theory, which forms a functional analytic background for the proofs of Theorems 1.1 and 1.2. In particular, we formulate a version of the HilleYosida theorem adapted to the present context (Theorem 3.1). Moreover, we give two useful criteria in order to prove that a linear operator be the infinitesimal generator of some Feller semigroup (Theorem 3.2 and Corollary 3.3). The results here are adapted from Bony-Courrège-Priouret [24] and Taira [13].

\subsection{Markov transition functions}

First, we give the precise definition of a transition function which is adapted to our analysis. From the viewpoint of functional analysis, the transition function is something more convenient than the Markov process itself.

Let $(K, \rho)$ be a locally compact, separable metric space and $\mathcal{B}$ the $\sigma$-algebra of all Borel sets in $K$. A function $p_{t}(x, E)$, defined for all $t \geq 0, x \in K$ and $E \in \mathcal{B}$, is called a (temporally homogeneous) Markov transition function on $K$ if it satisfies the following four conditions (a), (b), (c) and (d):

(a) $p_{t}(x, \cdot)$ is a non-negative measure on $\mathcal{B}$ and $p_{t}(x, K) \leq 1$ for each $t \geq 0$ and $x \in K$.

(b) $p_{t}(\cdot, E)$ is a Borel measurable function for each $t \geq 0$ and $E \in \mathcal{B}$.

(c) $p_{0}(x,\{x\})=1$ for each $x \in K$.

(d) (The Chapman-Kolmogorov equation) For any $t, s \geq 0, x \in K$ and $E \in \mathcal{B}$, we have the formula

$$
p_{t+s}(x, E)=\int_{K} p_{t}(x, d y) p_{s}(y, E) .
$$

It should be emphasized that equation (3.1) expresses the idea that a transition from the position $x$ to the set $E$ in time $t+s$ is composed of a transition from $x$ to some position $y$ in time $t$, followed by a transition from $y$ to the set $E$ in the remaining time $s$; the latter transition has probability $p_{s}(y, E)$ which depends only on $y$.

We add a point $\partial$ to the locally compact space $K$ as the point at infinity if $K$ is not compact, and as an isolated point if $K$ is compact; so the space $K_{\partial}=K \cup\{\partial\}$ is compact. 
Let $C(K)$ be the space of real-valued, bounded continuous functions $f$ on $K$. The space $C(K)$ is a Banach space with the supremum norm

$$
\|f\|=\sup _{x \in K}|f(x)|
$$

We introduce a closed subspace of $C(K)$ as follows:

$$
C_{0}(K)=\left\{f \in C(K): \lim _{x \rightarrow \partial} f(x)=0\right\} .
$$

It should be noticed that the space $C_{0}(K)$ may be identified with the subspace of $C\left(K_{\partial}\right)$ which consists of all functions $f$ satisfying $f(\partial)=0$ :

$$
C_{0}(K)=\left\{f \in C\left(K_{\partial}\right): f(\partial)=0\right\}
$$

Furthermore, we can extend a Markov transition function $p_{t}(x, \cdot)$ on $K$ to a Markov transition function $p_{t}^{\prime}(x, \cdot)$ on $K_{\partial}$ as follows:

$$
\begin{aligned}
& p_{t}^{\prime}(x, E)=p_{t}(x, E), x \in K, E \in \mathcal{B} \\
& p_{t}^{\prime}(x,\{\partial\})=1-p_{t}(x, K), x \in K \\
& p_{t}^{\prime}(\partial, K)=0, p_{t}^{\prime}(\partial,\{\partial\})=1 .
\end{aligned}
$$

Intuitively, this means that a Markovian particle moves in the space $K$ until it "dies" at which time it reaches the point $\partial$; hence the point $\partial$ is called the terminal point.

\subsection{Feller Semigroups and the Hille-Yosida Theorem}

We can associate with each Markov transition function $p_{t}(x, \cdot)$ a family $\left\{T_{t}\right\}_{t \geq 0}$ of bounded linear operators acting on the space $C_{0}(K)$, defined by the formula

$$
T_{t} f(x)=\int_{K} p_{t}(x, d y) f(y), \quad f \in C_{0}(K),
$$

and the Chapman-Kolmogorov equation (3.1) implies that this family $\left\{T_{t}\right\}_{t \geq 0}$ forms a semigroup.

A family $\left\{T_{t}\right\}_{t \geq 0}$ of bounded linear operators acting on $C_{0}(K)$ is called a Feller semigroup on $K$ if it satisfies the following three conditions (i), (ii) and (iii):

(i) $T_{t+s}=T_{t} \cdot T_{s}, t, s \geq 0 ; T_{0}=I$.

(ii) The family $\left\{T_{t}\right\}$ is strongly continuous in $t$ for each $t \geq 0$ :

$$
\lim _{s \downarrow 0}\left\|T_{t+s} f-T_{t} f\right\|=0, \quad f \in C_{0}(K) .
$$

(iii) The family $\left\{T_{t}\right\}$ is non-negative and contractive on $C_{0}(K)$ :

$$
f \in C_{0}(K), 0 \leq f(x) \leq 1 \quad \text { on } K \Longrightarrow 0 \leq T_{t} f(x) \leq 1 \quad \text { on } K \text {. }
$$


If $\left\{T_{t}\right\}_{t \geq 0}$ is a Feller semigroup on $K$, we define its infinitesimal generator $\mathfrak{A}$ by the formula

$$
\mathfrak{A} u=\lim _{t \downarrow 0} \frac{T_{t} u-u}{t},
$$

provided that the limit (3.2) exists in $C_{0}(K)$. More precisely, the infinitesimal generator $\mathfrak{A}$ is a linear operator from the space $C_{0}(K)$ into itself defined as follows.

(1) The domain $D(\mathfrak{A})$ is the set

$$
D(\mathfrak{A})=\left\{u \in C_{0}(K): \text { the limit (3.2) exists }\right\} .
$$

(2) $\mathfrak{A} u=\lim _{t \downarrow 0} \frac{T_{t} u-u}{t}, u \in D(\mathfrak{A})$.

The next theorem is a version of the Hille-Yosida theorem [25] adapted to the present context (see [13, Theorem 9.3.1 and Corollary 9.3.2]):

Theorem 3.1. (i) Let $\left\{T_{t}\right\}_{t>0}$ be a Feller semigroup on $K$ and $\mathfrak{A}$ its infinitesimal generator. Then we have the following four assertions (a), (b), (c) and $(d)$ :

(a) The domain $D(\mathfrak{A})$ is dense in the space $C_{0}(K)$.

(b) For each $\alpha>0$, the equation $(\alpha I-\mathfrak{A}) u=f$ has a unique solution $u$ in $D(\mathfrak{A})$ for any $f \in C_{0}(K)$. Hence, for each $\alpha>0$, the Green operator $(\alpha I-\mathfrak{A})^{-1}: C_{0}(K) \rightarrow C_{0}(K)$ can be defined by the formula

$$
u=(\alpha I-\mathfrak{A})^{-1} f, \quad f \in C_{0}(K) .
$$

(c) For each $\alpha>0$, the operator $(\alpha I-\mathfrak{A})^{-1}$ is non-negative on the space $C_{0}(K)$ :

$$
f \in C_{0}(K), f \geq 0 \quad \text { on } K \Longrightarrow(\alpha I-\mathfrak{A})^{-1} f \geq 0 \quad \text { on } K .
$$

(d) For each $\alpha>0$, the operator $(\alpha I-\mathfrak{A})^{-1}$ is bounded on the space $C_{0}(K)$ with norm

$$
\left\|(\alpha I-\mathfrak{A})^{-1}\right\| \leq \frac{1}{\alpha} .
$$

(ii) Conversely, if $\mathfrak{A}$ is a linear operator from $C_{0}(K)$ into itself satisfying condition (a) and if there is a constant $\alpha_{0} \geq 0$ such that, for all $\alpha>\alpha_{0}$, conditions (b) through (d) are satisfied, then $\mathfrak{A}$ is the infinitesimal generator of some Feller semigroup $\left\{T_{t}\right\}_{t \geq 0}$ on $K$.

Following [13], we recall two useful criteria in terms of the maximum principle in order that a linear operator be the infinitesimal generator of some Feller semigroup (see [13, Theorem 9.3.3 and Corollary 9.3.4]):

Theorem 3.2 (Hille-Yosida-Ray). Let $K$ be a compact metric space. Then we have the following two assertions (i) and (ii): 
(i) Let $B$ be a linear operator from $C(K)=C_{0}(K)$ into itself, and assume that the following two conditions $(\alpha)$ and $(\beta)$ are satisfied:

$(\alpha)$ The domain $D(B)$ of $B$ is dense in $C(K)$.

( $\beta)$ There exists an open and dense subset $K_{0}$ of $K$ such that if $u \in D(B)$ takes a positive maximum at a point $x_{0}$ of $K_{0}$, then we have the inequality

$$
B u\left(x_{0}\right) \leq 0 .
$$

Then the operator $B$ is closable in $C(K)$.

(ii) Let $B$ be as in part (i), and further assume that the following two conditions $\left(\beta^{\prime}\right)$ and $(\gamma)$ are satisfied:

$\left(\beta^{\prime}\right)$ If $u \in D(B)$ takes a positive maximum at a point $x^{\prime}$ of $K$, then we have the inequality

$$
B u\left(x^{\prime}\right) \leq 0
$$

$(\gamma)$ For some $\alpha_{0} \geq 0$, the range $R\left(\alpha_{0} I-B\right)$ of $\alpha_{0} I-B$ is dense in $C(K)$.

Then the minimal closed extension $\bar{B}$ of $B$ is the infinitesimal generator of some Feller semigroup on $K$.

Corollary 3.3. Let $A$ be the infinitesimal generator of a Feller semigroup on a compact metric space $K$ and let $B$ be a bounded linear operator on $C(K)$ into itself. If either $B$ or $C=A+B$ satisfies condition $\left(\beta^{\prime}\right)$ of Theorem 3.2, then the operator $C$ is the infinitesimal generator of some Feller semigroup on $K$.

\section{The Dirichlet Problem}

In this section we consider the Dirichlet problem for the diffusion operator with VMO coefficients in the framework of Sobolev spaces of $L^{p}$ style, and prove an existence and uniqueness theorem for the Dirichlet problem (Theorem 4.1). The uniqueness result in Theorem 4.1 follows from a variant of the Bakel'manAleksandrov maximum principle in the framework of Sobolev spaces, essentially due to Bony [16] (Theorem 4.4). The function spaces we shall treat here are the Sobolev spaces $W^{k, p}(\Omega)$ and the Besov spaces $B^{k-1 / p, p}(\partial \Omega)$ which enter naturally in connection with elliptic boundary value problems in the framework of Sobolev spaces of $L^{p}$ style. For more thorough treatments of this subject, the reader might be referred to Adams-Fournier [26], Bergh-Löfström [27] and Triebel [28].

\subsection{Formulation of the Dirichlet Problem}

An open set $\Omega$ in $\mathbf{R}^{N}$ is said to be of class $C^{1,1}$ if its boundary $\partial \Omega$ can be locally represented as the graph of a $C^{1}$ function whose first-order partial derivatives are all Lipschitz continuous. It should be emphasized that VMO functions are invariant under $C^{1,1}$-diffeomorphisms (see [23, Proposition 1.3]).

Let $\Omega$ be a bounded domain in Euclidean space $\mathbf{R}^{N}, N \geq 3$, with boundary $\partial \Omega$ of class $C^{1,1}$. If $1<p<\infty$ and if $k=1$ or $k=2$, we define the Sobolev space

$$
W^{2, p}(\Omega)=\text { the space of (equivalence classes of) functions }
$$


$u \in L^{p}(\Omega)$ whose derivatives $D^{\alpha} u,|\alpha| \leq 2$, in the sense of distributions are in $L^{p}(\Omega)$,

and the boundary space

$$
\begin{aligned}
B^{2-1 / p, p}(\partial \Omega)= & \text { the space of the boundary values } \gamma_{0} u \text { of functions } \\
& u \in W^{2, p}(\Omega) .
\end{aligned}
$$

In the boundary space $B^{2-1 / p, p}(\partial \Omega)$, we introduce a norm

$$
|\varphi|_{B^{2-1 / p, p}(\partial \Omega)}=\inf \left\{\|u\|_{W^{2, p}(\Omega)}: u \in W^{2, p}(\Omega), \gamma_{0} u=\varphi \text { on } \partial \Omega\right\} .
$$

It is known (see [26], [27], [28]) that the space $B^{2-1 / p, p}(\partial \Omega)$ is a Besov space.

Moreover, it should be emphasized (see [26, Theorem 5.37]) that the closure $W_{0}^{1, p}(\Omega)$ of $C_{0}^{\infty}(\Omega)$ in $W^{1, p}(\Omega)$ may be characterized as follows:

$$
W_{0}^{1, p}(\Omega)=\left\{u \in W^{1, p}(\Omega): \gamma_{0} u=0 \quad \text { on } \partial \Omega\right\} .
$$

In this subsection we consider the following non-homogeneous Dirichlet problem: Given functions $f(x)$ and $\varphi\left(x^{\prime}\right)$ defined in $\Omega$ and on $\partial \Omega$, respectively, find a function $u(x)$ in $\Omega$ such that

$$
\begin{cases}A u=f & \text { in } \Omega, \\ \gamma_{0} u=\varphi & \text { on } \partial \Omega .\end{cases}
$$

The next theorem is a generalization of Bony [16, Théorème 3] to the $V M O$ case:

Theorem 4.1. Let $N<p<\infty$, and assume that $c(x) \leq 0$ for almost all $x \in \Omega$. Then, for any $f \in L^{p}(\Omega)$ and any $\varphi \in B^{2-1 / p, p}(\partial \Omega)$, there exists a unique solution $u \in W^{2, p}(\Omega)$ of the Dirichlet problem (4.1).

If we associate with problem (4.1) a continuous linear operator

$$
\mathcal{A}=\left(A, \gamma_{0}\right): W^{2, p}(\Omega) \longrightarrow L^{p}(\Omega) \bigoplus B^{2-1 / p, p}(\partial \Omega),
$$

then we obtain from Theorem 4.1 that the mapping $\mathcal{A}$ is an algebraic and topological isomorphism. Indeed, the continuity of the inverse of $\mathcal{A}$ follows immediately from an application of Banach's open mapping theorem.

\subsection{Proof of Theorem 4.1}

The proof of Theorem 4.1 is divided into four steps.

Step 1: Our proof is essentially based on the following existence and uniqueness theorem for the homogeneous Dirichlet problem due to Chiarenza-FrascaLongo [8, Theorems 4.3 and 4.4] (see also [7]):

Theorem 4.2. Let $1<p<\infty$ and

$$
A_{0} u=\sum_{i, j=1}^{N} a^{i j}(x) \frac{\partial^{2} u}{\partial x_{i} \partial x_{j}} .
$$


Then, for any $f \in L^{p}(\Omega)$ with $1<p<\infty$ there exists a unique solution $u \in$ $W^{2, p}(\Omega)$ of the Dirichlet problem

$$
\begin{cases}A_{0} u=f & \text { in } \Omega, \\ \gamma_{0} u=0 & \text { on } \partial \Omega .\end{cases}
$$

Now, for any $\varphi \in B^{2-1 / p, p}(\partial \Omega)$, we can find a function $v \in W^{2, p}(\Omega)$ such that $\gamma_{0} v=\varphi$ on $\partial \Omega$ (see [26, Theorem 7.39]). Hence we obtain the following existence and uniqueness theorem for the non-homogeneous Dirichlet problem:

Corollary 4.3. Let $1<p<\infty$. For any $f \in L^{p}(\Omega)$ and any $\varphi \in B^{2-1 / p, p}(\partial \Omega)$, there exists a unique solution $u \in W^{2, p}(\Omega)$ of the Dirichlet problem

$$
\begin{cases}A_{0} u=f & \text { in } \Omega, \\ \gamma_{0} u=\varphi & \text { on } \partial \Omega .\end{cases}
$$

We recall that a linear operator $T$ from a Banach space $X$ into a Banach space $Y$ is called a Fredholm operator if it satisfies the following five conditions (i) through (v):

(i) The domain $D(T)$ of $T$ is dense in $X$.

(ii) $T$ is a closed operator.

(iii) The null space $N(T)=\{x \in D(T): T x=0\}$ of $T$ has finite dimension; $\operatorname{dim} N(T)<\infty$.

(iv) The range $R(T)=\{T x: x \in D(T)\}$ of $T$ is closed in $Y$.

(v) The range $R(T)$ of $T$ has finite codimension; $\operatorname{codim} R(T)=\operatorname{dim} Y / R(T)<$ $\infty$.

Then the index of $T$ is defined by the formula

$$
\text { ind } T:=\operatorname{dim} N(T)-\operatorname{codim} R(T) \text {. }
$$

If we associate with problem (4.3) a continuous linear operator

$$
\mathcal{A}_{0}=\left(A_{0}, \gamma_{0}\right): W^{2, p}(\Omega) \longrightarrow L^{p}(\Omega) \bigoplus B^{2-1 / p, p}(\partial \Omega),
$$

then Corollary 4.3 asserts that the mapping $\mathcal{A}_{0}$ is an algebraic and topological isomorphism. In particular, we have, for $1<p<\infty$,

$$
\text { ind } \mathcal{A}_{0}=0 \text {. }
$$

Step 2: If we let

$$
B u=\sum_{i=1}^{N} b^{i}(x) \frac{\partial u}{\partial x_{i}}+c(x) u,
$$

then it is clear that the operator

$$
B: W^{2, p}(\Omega) \longrightarrow W^{1, p}(\Omega)
$$


is continuous. However, it follows from an application of the Rellich and Kondrachov theorem (see $[1$, Theorem 7.26$]$ ) that the injection $W^{1, p}(\Omega) \rightarrow L^{p}(\Omega)$ is compact. Hence we find that the mapping

$$
B: W^{2, p}(\Omega) \longrightarrow L^{p}(\Omega)
$$

is compact.

Therefore, we obtain that the mapping

$$
\mathcal{A}=\left(A_{0}+B, \gamma_{0}\right)=\mathcal{A}_{0}+(B, 0): W^{2, p}(\Omega) \longrightarrow L^{p}(\Omega) \bigoplus B^{2-1 / p, p}(\partial \Omega)
$$

is a Fredholm operator with index zero, since we have, by assertion (4.4),

$$
\text { ind } \mathcal{A}=\text { ind } \mathcal{A}_{0}=0 \text {. }
$$

Step 3: On the other hand, the uniqueness result in Theorem 4.1 follows from a variant of the Bakel'man-Aleksandrov maximum principle in the framework of Sobolev spaces due to Bony [16, Théorème 2]:

Theorem 4.4. Assume that $c(x) \leq 0$ for almost all $x \in \Omega$. If a function $u \in W^{2, p}(\Omega), N<p<\infty$, satisfies the condition

$$
A u(x) \geq 0 \text { for almost all } x \in \Omega,
$$

then we have the inequality

$$
\max _{\bar{\Omega}} u \leq \max _{\partial \Omega} u^{+}
$$

where

$$
u^{+}(x)=\max \{u(x), 0\}, \quad x \in \bar{\Omega} .
$$

Remark 4.1. More precisely, Bony proved this maximum principle under the weaker condition that $a^{i j} \in L^{\infty}(\Omega)$ (see the proof of [16, Théorème 1]).

By applying Theorem 4.4 to the functions $\pm u(x)$, we obtain that

$$
\left\{\begin{array}{ll}
A u=0 & \text { almost everywhere in } \Omega, \\
\gamma_{0} u=0 & \text { on } \partial \Omega
\end{array} \quad u=0 \quad \text { in } \Omega .\right.
$$

This proves that the mapping

$$
\mathcal{A}=\left(A, \gamma_{0}\right): W^{2, p}(\Omega) \longrightarrow L^{p}(\Omega) \bigoplus B^{2-1 / p, p}(\partial \Omega)
$$

is injective for $N<p<\infty$. Hence it is also surjective for $N<p<\infty$, since we have

$$
\text { ind } \mathcal{A}=\operatorname{dim} N(\mathcal{A})-\operatorname{codim} R(\mathcal{A})=0
$$

Step 4: Summing up, we have proved that the mapping

$$
\mathcal{A}=\left(A, \gamma_{0}\right): W^{2, p}(\Omega) \longrightarrow L^{p}(\Omega) \bigoplus B^{2-1 / p, p}(\partial \Omega)
$$

is an algebraic and topological isomorphism for $N<p<\infty$.

Now the proof of Theorem 4.1 is complete. 


\section{The Oblique Derivative Case}

In this section we study the oblique derivative problem in the framework of Sobolev spaces of $L^{p}$ style, and prove an existence and uniqueness theorem for the oblique derivative problem with VMO coefficients (Theorem 5.1). The uniqueness result in Theorem 5.1 follows from a variant of the Bakel'manAleksandrov maximum principle in the framework of Sobolev spaces due to Lieberman [17] (Theorem 5.3). Moreover, we construct a Feller semigroup associated with absorption, reflection and drift phenomena at the boundary (Theorem 1.2).

\subsection{Formulation of the Oblique Derivative Problem}

In this section, we consider an oblique derivative boundary operator of the form

$$
L_{0} u:=\mu\left(x^{\prime}\right) \frac{\partial u}{\partial \mathbf{n}}+\beta\left(x^{\prime}\right) \cdot u+\gamma\left(x^{\prime}\right) u .
$$

The purpose of this subsection is to prove an existence and uniqueness theorem for the following non-homogeneous oblique derivative problem in the framework of Sobolev spaces of $L^{p}$ style:

$$
\begin{cases}A u=f & \text { in } \Omega \\ L_{0} u=\varphi & \text { on } \partial \Omega .\end{cases}
$$

Our starting point is the following existence and uniqueness theorem for the non-homogeneous oblique derivative problem with $V M O$ coefficients:

Theorem 5.1. Let $N<p<\infty$ and $\alpha \geq 0$. Assume that conditions (H.1) and (H.2) are satisfied. Then, for any $f \in L^{p}(\Omega)$ and any $\varphi \in B^{1-1 / p, p}(\partial \Omega)$ the non-homogeneous oblique derivative problem

$$
\begin{cases}(A-\alpha) u=f & \text { in } \Omega, \\ L_{0} u=\varphi & \text { on } \partial \Omega\end{cases}
$$

has a unique solution $u \in W^{2, p}(\Omega)$.

If we associate with problem (5.2) a continuous linear operator

$$
\mathcal{A}_{N}(\alpha)=\left(A-\alpha, L_{0}\right): W^{2, p}(\Omega) \longrightarrow L^{p}(\Omega) \oplus B^{1-1 / p, p}(\partial \Omega),
$$

then we obtain from the trace theorem (see [26, Remarks 7.45]) and Theorem 5.1 that the mapping $\mathcal{A}_{N}(\alpha)$ is an algebraic and topological isomorphism, for any $\alpha \geq 0$. Indeed, the continuity of the inverse of $\mathcal{A}_{N}(\alpha)$ follows immediately from an application of Banach's open mapping theorem.

\subsection{Proof of Theorem 5.1}

The proof of Theorem 5.1 is divided into four steps. First, since $\mathbf{n}$ is the unit interior normal to the boundary $\partial \Omega$, it follows that

$$
\left\langle\mu\left(x^{\prime}\right) \mathbf{n}+\beta\left(x^{\prime}\right), \mathbf{n}\right\rangle=\mu\left(x^{\prime}\right)\langle\mathbf{n}, \mathbf{n}\rangle=\mu\left(x^{\prime}\right), \quad x^{\prime} \in \partial \Omega .
$$


Therefore, we find that condition (H.1) is equivalent to the condition that the directional derivative associated with $L_{0}$ is nowhere tangential to the boundary $\partial \Omega$.

Step 1: Our proof is based on the following existence and uniqueness theorem for the non-homogeneous oblique derivative problem (see [29, Theorem 4.1], [30]):

Theorem 5.2. Let $1<p<\infty, \alpha \geq 0$ and

$$
A_{0} u=\sum_{i, j=1}^{N} a^{i j}(x) \frac{\partial^{2} u}{\partial x_{i} \partial x_{j}} .
$$

Assume that conditions (H.1) and (H.2) are satisfied. Then, for any $f \in L^{p}(\Omega)$ and any $\varphi \in B^{1-1 / p, p}(\partial \Omega)$ there exists a unique solution $u \in W^{2, p}(\Omega)$ of the oblique derivative problem

$$
\begin{cases}\left(A_{0}-\alpha\right) u=f & \text { in } \Omega, \\ L_{0} u=\varphi & \text { on } \partial \Omega .\end{cases}
$$

If we associate with problem (5.3) a continuous linear operator

$$
\mathcal{A}_{0}(\alpha)=\left(A_{0}-\alpha, L_{0}\right): W^{2, p}(\Omega) \longrightarrow L^{p}(\Omega) \oplus B^{1-1 / p, p}(\partial \Omega),
$$

then we obtain from the trace theorem (see [26, Remarks 7.45]) and Theorem 5.2 that the mapping $\mathcal{A}_{0}(\alpha)$ is an algebraic and topological isomorphism, for any $\alpha \geq 0$. In particular, we have, for all $\alpha \geq 0$,

$$
\text { ind } \mathcal{A}_{0}=\operatorname{dim} N\left(\mathcal{A}_{0}\right)-\operatorname{codim} R\left(\mathcal{A}_{0}\right)=0 .
$$

Step 2: If we let

$$
\mathcal{B}(\alpha) u:=\sum_{i=1}^{N} b^{i}(x) \frac{\partial u}{\partial x_{i}}+(c(x)-\alpha) u, \quad \alpha \geq 0,
$$

then it follows that the operator

$$
\mathcal{B}(\alpha): W^{2, p}(\Omega) \longrightarrow W^{1, p}(\Omega)
$$

is continuous. However, it follows from an application of the Rellich and Kondrachov theorem (see $[1$, Theorem 7.26$]$ ) that the injection $W^{1, p}(\Omega) \rightarrow L^{p}(\Omega)$ is compact. Hence we find that the mapping

$$
\mathcal{B}(\alpha): W^{2, p}(\Omega) \longrightarrow L^{p}(\Omega)
$$

is compact. It should be noticed that

$$
\mathcal{A}_{N}(\alpha)=\left(A-\alpha, L_{0}\right)=\left(A_{0}-\alpha, L_{0}\right)+(\mathcal{B}(\alpha), 0)=\mathcal{A}_{0}(\alpha)+(\mathcal{B}(\alpha), 0) .
$$

Therefore, we obtain that the mapping

$$
\mathcal{A}_{N}(\alpha)=\mathcal{A}_{0}(\alpha)+(\mathcal{B}(\alpha), 0): W^{2, p}(\Omega) \longrightarrow L^{p}(\Omega) \oplus B^{1-1 / p, p}(\partial \Omega)
$$


is a Fredholm operator with index zero, for any $\alpha \geq 0$, since we have, by Theorem and assertion (5.4),

$$
\operatorname{ind} \mathcal{A}_{N}(\alpha)=\operatorname{ind} \mathcal{A}_{0}(\alpha)=0 \text {. }
$$

Step 3: On the other hand, the uniqueness result in Theorem 5.1 follows from an application of the Bakel'man and Aleksandrov maximum principle (see [17, Corollary 2.4]):

Theorem 5.3. Let $N<p<\infty$ and $\alpha \geq 0$. Assume that conditions (H.1) and (H.2) are satisfied. If a function $u \in W^{2, p}(\Omega)$ satisfies the conditions

$$
\begin{cases}(A-\alpha) u \leq 0 & \text { almost everywhere in } \Omega, \\ L_{0} u \leq 0 & \text { on } \partial \Omega,\end{cases}
$$

then it follows that either $u(x)$ is a non-negative constant function or $u(x)>0$ on $\bar{\Omega}$.

Proof. First, it should be noticed that we have, by Sobolev's imbedding theorem (see [26, Theorem 4.12, Part II]),

$$
W^{2, p}(\Omega) \subset C^{1}(\bar{\Omega}),
$$

since $2-N / p>1$ for $N<p<\infty$.

We have only to consider the case where $u(x)$ is not a constant function in $\Omega$. We assume, to the contrary, that $u(x)$ takes a non-positive minimum at a point $x_{0} \in \bar{\Omega}$. If we let

$$
v(x)=-u(x)
$$

then we have

$$
\begin{cases}v \in W^{2, p}(\Omega), \quad N<p<\infty, & \\ (A-\alpha) v=-(\alpha-A) u \geq 0 & \text { almost everywhere in } \Omega, \\ L_{0} v \geq 0 & \text { on } \partial \Omega .\end{cases}
$$

Hence, by applying the strong maximum principle to the function $v(x)$ we obtain that, for some boundary point $x_{0}^{\prime} \in \partial \Omega$,

$$
v\left(x_{0}^{\prime}\right)=v\left(x_{0}\right)=\max _{\bar{\Omega}}(-u) \geq 0 .
$$

Moreover, it follows from an application of the Hopf boundary point lemma (Lemma A.2) that

$$
\frac{\partial v}{\partial \mathbf{n}}\left(x_{0}^{\prime}\right)<0 .
$$

By conditions (H.1) and (H.2), this implies that

$$
0 \leq L_{0} v\left(x_{0}^{\prime}\right)=\mu\left(x_{0}^{\prime}\right) \frac{\partial u}{\partial \mathbf{n}}\left(x_{0}^{\prime}\right)+\gamma\left(x_{0}^{\prime}\right) u\left(x_{0}^{\prime}\right) \leq \mu\left(x_{0}^{\prime}\right) \frac{\partial u}{\partial \mathbf{n}}\left(x_{0}^{\prime}\right)<0 .
$$

This contradiction proves that $u(x)>0$ on $\bar{\Omega}$.

The proof of Theorem 5.3 is complete. 
By applying Theorem 5.3 to the functions $\pm u(x)$, it follows that we have, for $\alpha \geq 0$,

$$
\left\{\begin{array}{ll}
(A-\alpha) u=0 & \text { almost everywhere in } \Omega, \\
L_{0} u=0 & \text { on } \partial \Omega
\end{array} \Longrightarrow u=0 \quad \text { in } \Omega .\right.
$$

This proves that the mapping

$$
\mathcal{A}_{N}(\alpha)=\left(A-\alpha, L_{0}\right): W^{2, p}(\Omega) \longrightarrow L^{p}(\Omega) \oplus B^{1-1 / p, p}(\partial \Omega)
$$

is injective for any $\alpha \geq 0$ if $N<p<\infty$. Hence it is also surjective for any $\alpha \geq 0$ if $N<p<\infty$, since we have, for any $\alpha \geq 0$,

$$
\text { ind } \mathcal{A}_{N}(\alpha)=\operatorname{dim} N\left(\mathcal{A}_{N}(\alpha)\right)-\operatorname{codim} R\left(\mathcal{A}_{N}(\alpha)\right)=0 .
$$

Step 4: Summing up, we have proved that the mapping

$$
\mathcal{A}_{N}(\alpha)=\left(A-\alpha, L_{0}\right): W^{2, p}(\Omega) \longrightarrow L^{p}(\Omega) \oplus B^{1-1 / p, p}(\partial \Omega)
$$

is an algebraic and topological isomorphism for any $\alpha \geq 0$ if $N<p<\infty$.

Now the proof of Theorem 5.1 is complete.

\subsection{Proof of Theorem $\mathbf{1 . 2}$}

In order to prove Theorem 1.2, it suffices to verify all conditions (a) through (d) in Theorem 3.1 with

$$
\begin{aligned}
& K:=\bar{\Omega}, \\
& C_{0}(K):=C(\bar{\Omega}), \\
& \mathfrak{A}:=\mathfrak{A}_{N} .
\end{aligned}
$$

The proof of Theorem 1.2 is divided into four steps.

Step 1: First, we prove that, for each $\alpha \geq 0$, the equation $(\alpha I-A) u=f$ has a unique solution $u \in D\left(\mathfrak{A}_{N}\right)$ for any $f \in C(\bar{\Omega})$.

By applying Theorem 5.1, we obtain that the oblique derivative problem

$$
\begin{cases}(\alpha-A) u=f & \text { almost everywhere in } \Omega, \\ L_{0} u=0 & \text { on } \partial \Omega\end{cases}
$$

has a unique solution $u \in W^{2, p}(\Omega)$ for any $f \in L^{p}(\Omega)$ with $N<p<\infty$. In particular, for any $f \in C(\bar{\Omega})$ there exists a function $u \in W^{2, p}(\Omega)$ such that

$$
(\alpha-A) u=f \quad \text { in } \Omega .
$$

Hence we have

$$
A u=\alpha u-f \in C(\bar{\Omega}) .
$$

By formula (1.4), this proves that

$$
\left\{\begin{array}{l}
u \in D\left(\mathfrak{A}_{N}\right) \\
\left(\alpha I-\mathfrak{A}_{N}\right) u=f .
\end{array}\right.
$$


Step 2: Secondly, we prove that, for each $\alpha \geq 0$, the Green operator $G_{\alpha}^{N}=$ $\left(\alpha I-\mathfrak{A}_{N}\right)^{-1}$ is non-negative on the space $C(\bar{\Omega})$ :

$$
f \in C(\bar{\Omega}), f(x) \geq 0 \quad \text { in } \Omega \Longrightarrow u(x)=G_{\alpha}^{N} f(x) \geq 0 \text { in } \Omega .
$$

More precisely, we prove the following assertion:

$$
f \in C(\bar{\Omega}), f(x) \geq 0, f(x) \not \equiv 0 \quad \text { in } \Omega \Longrightarrow u(x)=G_{\alpha}^{N} f(x)>0 \quad \text { on } \bar{\Omega} .
$$

Since we have

$$
\begin{cases}u \in W^{2, p}(\Omega), \quad N<p<\infty, & \\ (A-\alpha) u=-f \leq 0 & \text { almost everywhere in } \Omega, \\ L_{0} u=0 & \text { on } \partial \Omega,\end{cases}
$$

by applying Theorem 5.3 we obtain that either $u(x)$ is a non-negative constant function or $u(x)>0$ on $\bar{\Omega}$. However, if $u(x) \equiv 0$ in $\Omega$, then it follows that

$$
f(x)=(\alpha-A) u(x) \equiv 0 \quad \text { in } \Omega .
$$

This contradiction proves that either $u(x)$ is a positive constant function or $u(x)>0$ on $\bar{\Omega}$, that is,

$$
G_{\alpha}^{N} f(x)>0 \quad \text { on } \bar{\Omega} .
$$

Step 3: Thirdly, we prove that, for each $\alpha>0$, the Green operator $G_{\alpha}^{N}=$ $\left(\alpha I-\mathfrak{A}_{N}\right)^{-1}$ is bounded on the space $C(\bar{\Omega})$ with norm $1 / \alpha$ : $\left\|G_{\alpha}^{N}\right\| \leq 1 / \alpha$.

By assertion (5.5), it suffices to show that

$$
\alpha G_{\alpha}^{N} 1(x) \leq 1 \quad \text { on } \bar{\Omega} .
$$

If we let

$$
v(x):=\alpha G_{\alpha}^{N} 1(x)-1,
$$

then we have

$$
\begin{cases}u \in W^{2, p}(\Omega), \quad N<p<\infty, & \\ (A-\alpha) v=0 & \text { in } \Omega, \\ L_{0} v=0 & \text { on } \partial \Omega .\end{cases}
$$

By applying Theorem 5.3 to the function $-v(x)$, we arrive at a contradiction that

$$
\max _{\bar{\Omega}} v>0 \Longrightarrow v(x) \equiv 0 \quad \text { in } \Omega .
$$

This proves that

$$
\max _{\bar{\Omega}} v \leq 0
$$

or equivalently,

$$
\alpha G_{\alpha}^{N} 1(x) \leq 1 \quad \text { on } \bar{\Omega} .
$$

Step 4: The closedness of $\mathfrak{A}_{N}$ is an immediate consequence of the boundedness of $G_{\alpha}^{N}=\left(\alpha I-\mathfrak{A}_{N}\right)^{-1}$. Indeed, it suffices to note the formula

$$
\mathfrak{A}_{N}=\alpha I-\left(G_{\alpha}^{N}\right)^{-1} .
$$


Step 5: Finally, we prove that the domain $D\left(\mathfrak{A}_{N}\right)$ is dense in $C(\bar{\Omega})$. More precisely, we prove that, for each $u \in C(\bar{\Omega})$, we have

$$
\lim _{\alpha \rightarrow+\infty}\left\|\alpha G_{\alpha}^{N} u-u\right\|_{C(\bar{\Omega})}=0 .
$$

Step 5-1:It suffices to prove assertion (5.6) for any $v \in C^{2}(\bar{\Omega})$ such that $L_{0} v=0$ on $\partial \Omega$. In fact, we have the following (see [31, Lemma 3.2]):

Lemma 5.4. Let $u \in C(\bar{\Omega})$. For any given $\varepsilon>0$, we can find a function $v \in C^{2}(\bar{\Omega})$ such that

$$
\left\{\begin{array}{l}
\|u-v\|_{C(\bar{\Omega})}<\varepsilon \\
L_{0} v=0 \quad \text { on } \partial \Omega .
\end{array}\right.
$$

Proof. First, it follows from an application of the Weierstrass approximation theorem that there exists a polynomial $g(x)$ such that

$$
\|u-g\|_{C(\bar{\Omega})}<\frac{\varepsilon}{2}
$$

Secondly, we can construct a function $h(x) \in C^{2}(\bar{\Omega})$ such that

$$
\begin{aligned}
& h=0 \quad \text { on } \partial \Omega, \\
& \frac{\partial h}{\partial \mathbf{n}}=\frac{1}{\mu\left(x^{\prime}\right)} L_{0} g \quad \text { on } \partial \Omega, \\
& \|h\|_{C(\bar{\Omega})}<\frac{\varepsilon}{2} .
\end{aligned}
$$

This implies that

$$
L_{0} h=\mu\left(x^{\prime}\right) \frac{\partial h}{\partial \mathbf{n}}+\beta\left(x^{\prime}\right) \cdot h+\gamma\left(x^{\prime}\right) h=\mu\left(x^{\prime}\right) \frac{\partial h}{\partial \mathbf{n}}=L_{0} g \quad \text { on } \partial \Omega .
$$

Therefore, it is easy to verify that the function $v(x)=g(x)-h(x)$ satisfies the desired conditions (5.7).

The proof of Lemma 5.4 is complete.

Step 5-2:To prove assertion (5.6) for any $v \in C^{2}(\bar{\Omega})$ such that $L_{0} v=0$, we introduce an extension $\widetilde{G_{\alpha}^{N}}$ of the Green operator $G_{\alpha}^{N}$ to the space $L^{p}(\Omega)$ for $N<p<\infty$ :

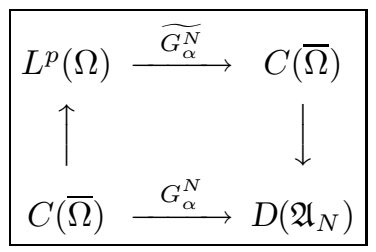

By Theorem 5.1, we find that the oblique derivative problem

$$
\begin{cases}(\alpha-A) u=f & \text { almost everywhere in } \Omega, \\ L_{0} u=0 & \text { on } \partial \Omega\end{cases}
$$

has a unique solution $u \in W^{2, p}(\Omega)$ for any $f \in L^{p}(\Omega)$. If we let

$$
u:=\widetilde{G_{\alpha}^{N}} f,
$$


then it is easy to verify that the operator $\widetilde{G_{\alpha}^{N}}$ is an extension of $G_{\alpha}^{N}$ to $L^{p}(\Omega)$. Moreover, just as in Steps 2 and 3 we can prove the following two assertions (A) and (B):

(A) The operator $\widetilde{G_{\alpha}^{N}}: L^{p}(\Omega) \rightarrow C(\bar{\Omega})$ is non-negative.

(B) The operator $\widetilde{G_{\alpha}^{N}}: L^{\infty}(\Omega) \rightarrow C(\bar{\Omega})$ is bounded with norm $1 / \alpha$ : $\left\|\widetilde{G_{\alpha}^{N}}\right\| \leq$ $1 / \alpha$.

First, since $a^{i j}(x), b^{i}(x), c(x) \in L^{\infty}(\Omega)$ and $v \in C^{2}(\bar{\Omega})$, it follows that

$$
A v=\sum_{i, j=1}^{N} a^{i j}(x) \frac{\partial^{2} v}{\partial x_{i} \partial x_{j}}+\sum_{i=1}^{N} b^{i}(x) \frac{\partial v}{\partial x_{i}}+c(x) v \in L^{\infty}(\Omega) .
$$

Thus, if we let

$$
w:=\alpha G_{\alpha}^{N} v+\widetilde{G_{\alpha}^{N}}(A v)
$$

then we have

$$
\left\{\begin{array}{l}
w \in W^{2, p}(\Omega) \cap C^{1}(\bar{\Omega}), \quad N<p<\infty, \\
(A-\alpha) w=(A-\alpha) v \quad \text { almost everywhere in } \Omega, \\
L_{0} w=0 \text { on } \partial \Omega,
\end{array}\right.
$$

and so

$$
\left\{\begin{array}{l}
w-v \in W^{2, p}(\Omega) \cap C(\bar{\Omega}), \quad N<p<\infty \\
(A-\alpha)(w-v)=0 \quad \text { almost everywhere in } \Omega, \\
L_{0}(w-v)=0 \quad \text { on } \partial \Omega .
\end{array}\right.
$$

By applying Theorem 5.1 to the function $w(x)-v(x)$, we obtain that $w-v=0$ in $\Omega$. This implies that

$$
v=w=\alpha G_{\alpha}^{N} v+\widetilde{G_{\alpha}^{N}}(A v) .
$$

Therefore, the desired assertion (5.6) for any $v \in C^{2}(\bar{\Omega})$ such that $L_{0} v=0$ follows from an application of assertion (B), since we have, for all $\alpha>0$,

$$
\left\|v-\alpha G_{\alpha}^{N} v\right\|_{C(\bar{\Omega})}=\left\|\widetilde{G_{\alpha}^{N}}(A v)\right\|_{C(\bar{\Omega})} \leq \frac{1}{\alpha}\|A v\|_{L^{\infty}(\Omega)} .
$$

Now the proof of Theorem 1.2 is complete.

\subsection{Proof of Remark 1.2}

Finally, we prove that the domain

$$
D\left(\mathfrak{A}_{N}\right)=\left\{u \in W^{2, p}(\Omega): A u \in C(\bar{\Omega}), L_{0} u=0 \text { on } \partial \Omega\right\}
$$

is independent of $N<p<\infty$.

We let

$$
\mathcal{D}_{p}:=\left\{u \in W^{2, p}(\Omega): A u \in C(\bar{\Omega}), L_{0} u=0 \text { on } \partial \Omega\right\} .
$$


In order to prove Remark 1.2 , it suffices to show that

$$
\mathcal{D}_{p_{1}}=\mathcal{D}_{p_{2}} \quad \text { for } N<p_{1}<p_{2}<\infty
$$

First, it follows that

$$
\mathcal{D}_{p_{2}} \subset \mathcal{D}_{p_{1}}
$$

since we have $L^{p_{2}}(\Omega) \subset L^{p_{1}}(\Omega)$ for $p_{2}>p_{1}$.

Conversely, let $v$ be an arbitrary element of $\mathcal{D}_{p_{1}}$ :

$$
v \in W^{2, p_{1}}(\Omega), \quad A v \in C(\bar{\Omega}), L_{0} u=0 \text { on } \partial \Omega .
$$

Then, since we have $v, A v \in C(\bar{\Omega}) \subset L^{p_{2}}(\Omega)$, it follows from an application of Theorem 5.1 with $p:=p_{2}$ that there exists a unique function $u \in W^{2, p_{2}}(\Omega)$ such that

$$
\begin{cases}(A-\alpha) u=(A-\alpha) v & \text { in } \Omega \\ L_{0} u=0 & \text { on } \partial \Omega .\end{cases}
$$

Hence we have

$$
\begin{cases}u-v \in W^{2, p_{1}}(\Omega), & \\ (A-\alpha)(u-v)=0 & \text { in } \Omega, \\ L_{0}(u-v)=0 & \text { on } \partial \Omega .\end{cases}
$$

Therefore, by applying again Theorem 5.1 with $p:=p_{1}$ we obtain that $u-v=0$, so that $v=u \in W^{2, p_{2}}(\Omega)$. This implies that $v \in \mathcal{D}_{p_{2}}$.

The proof of Remark 1.2 is complete.

\section{Feller Semigroups and Boundary Value Prob- lems}

The purpose of this section is to prove a general existence theorem for Feller semigroups in terms of boundary value problems (Theorem 6.9), following the main idea of Taira [13, Section 9.6] (see Bony-Courrège-Priouret [24] and SatoUeno [32]). Intuitively, Theorem 6.9 asserts that we can "piece together" a Markov process on the boundary $\partial \Omega$ with $A$-diffusion in the interior $\Omega$ to construct a Markov process on the closure $\bar{\Omega}=\Omega \cup \partial \Omega$.

\subsection{Green Operators and Harmonic Operators}

Let $N<p<\infty$ and $\alpha>0$. Since we have

$$
c(x)-\alpha \leq-\alpha \text { for almost all } x \in \Omega,
$$

by applying Theorem 4.1 to the operator $A-\alpha$ we obtain that, for any $f \in C(\bar{\Omega})$ and any $\varphi \in C^{2}(\partial \Omega)$ the Dirichlet problem

$$
\begin{cases}(\alpha-A) u=f & \text { almost everywhere in } \Omega, \\ u=\varphi & \text { on } \partial \Omega\end{cases}
$$

has a unique solution $u \in W^{2, p}(\Omega)$, since $C(\bar{\Omega}) \subset L^{p}(\Omega)$ and $C^{2}(\partial \Omega) \subset$ $B^{2-1 / p, p}(\partial \Omega)$. Therefore, we can introduce two linear operators

$$
G_{\alpha}^{0}: C(\bar{\Omega}) \longrightarrow C(\bar{\Omega})
$$


and

$$
H_{\alpha}: C^{2}(\partial \Omega) \longrightarrow C(\bar{\Omega})
$$

as follows.

(a) For any $f \in C(\bar{\Omega})$, the function $G_{\alpha}^{0} f \in W^{2, p}(\Omega) \cap W_{0}^{1, p}(\Omega)$ is the unique solution of the problem

$$
\begin{cases}(\alpha-A) G_{\alpha}^{0} f=f & \text { in } \Omega, \\ G_{\alpha}^{0} f=0 & \text { on } \partial \Omega .\end{cases}
$$

(b) For any $\varphi \in C^{2}(\partial \Omega)$, the function $H_{\alpha} \varphi \in W^{2, p}(\Omega)$ is the unique solution of the problem

$$
\begin{cases}(\alpha-A) H_{\alpha} \varphi=0 & \text { in } \Omega, \\ H_{\alpha} \varphi=\varphi & \text { on } \partial \Omega .\end{cases}
$$

The operators $G_{\alpha}^{0}: C(\bar{\Omega}) \rightarrow C(\bar{\Omega})$ and $H_{\alpha}: C^{2}(\partial \Omega) \rightarrow C(\bar{\Omega})$ can be visualized as follows:
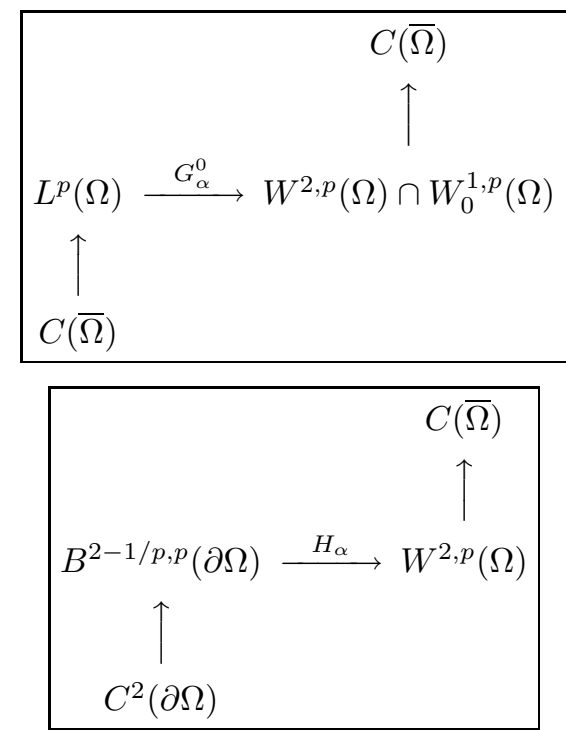

Here it should be noticed that we have, by Sobolev's imbedding theorem (see [26, Theorem 4.12, Part II]),

$$
W^{2, p}(\Omega) \subset C^{1}(\bar{\Omega}),
$$

and, by an imbedding theorem for Besov spaces (see [26, Theorem 7.34]),

$$
C^{2}(\partial \Omega) \subset B^{2-1 / p, p}(\partial \Omega) \subset C^{1}(\partial \Omega),
$$

since $2-N / p>1$ and $(1-1 / p) p=p-1>N-1$ for $N<p<\infty$.

The operator $G_{\alpha}^{0}$ is called the Green operator and the operator $H_{\alpha}$ is called the harmonic operator.

Then we have the following fundamental results for the operators $G_{\alpha}^{0}$ and $H_{\alpha}$ : 
Theorem 6.1. (i) (a) The Green operators $G_{\alpha}^{0}, \alpha>0$, are non-negative and bounded with norm

$$
\left\|G_{\alpha}^{0}\right\|=\left\|G_{\alpha}^{0} 1\right\|_{C(\bar{\Omega})} \leq \frac{1}{\alpha}
$$

(b) For any $f \in C(\bar{\Omega})$, we have the assertion

$$
G_{\alpha}^{0} f=0 \quad \text { on } \partial \Omega \text {. }
$$

(c) For all $\alpha, \beta>0$, the resolvent equation holds:

$$
G_{\alpha}^{0} f-G_{\beta}^{0} f+(\alpha-\beta) G_{\alpha}^{0}\left(G_{\beta}^{0} f\right)=0, \quad f \in C(\bar{\Omega}) .
$$

(d) For any $f \in C(\bar{\Omega})$, we have the assertion

$$
\lim _{\alpha \rightarrow+\infty} \alpha G_{\alpha}^{0} f(x)=f(x), \quad x \in \Omega .
$$

Furthermore, if $\left.f\right|_{\partial \Omega}=0$, that is, if $f \in C_{0}(\bar{\Omega})$, then this convergence is uniform in $x \in \bar{\Omega}$. In other words, we have the assertion

$$
\lim _{\alpha \rightarrow+\infty} \alpha G_{\alpha}^{0} f=f \quad \text { in } C_{0}(\bar{\Omega}) .
$$

(ii) (e) The harmonic operators $H_{\alpha}, \alpha>0$, can be uniquely extended to non-negative, bounded linear operators on $C(\partial \Omega)$ into $C(\bar{\Omega})$, denoted again by $H_{\alpha}$, with norm $\left\|H_{\alpha}\right\|=1$.

(f) For any $\varphi \in C(\partial \Omega)$, we have the assertion

$$
H_{\alpha} \varphi=\varphi \quad \text { on } \partial \Omega \text {. }
$$

(g) For all $\alpha, \beta>0$, we have the equation

$$
H_{\alpha} \varphi-H_{\beta} \varphi+(\alpha-\beta) G_{\alpha}^{0}\left(H_{\beta} \varphi\right)=0, \quad \varphi \in C(\partial \Omega)
$$

Proof. (i) Assertion (a): First, we show that the operators $G_{\alpha}^{0}$ are non-negative for all $\alpha>0$ :

$$
f \in C(\bar{\Omega}), f(x) \geq 0 \quad \text { in } \Omega \Longrightarrow G_{\alpha}^{0} f(x) \geq 0 \quad \text { in } \Omega .
$$

If we let

$$
v(x):=-G_{\alpha}^{0} f(x),
$$

then it follows that

$$
\begin{cases}(A-\alpha) v=f \geq 0 & \text { in } \Omega \\ \gamma_{0} v=0 & \text { on } \partial \Omega .\end{cases}
$$

Therefore, by applying the weak maximum principle (Theorem 4.4) with $A:=$ $A-\alpha$ we obtain that

$$
v(x) \leq 0 \quad \text { in } \Omega,
$$

so that

$$
G_{\alpha}^{0} f(x)=-v(x) \geq 0 \quad \text { in } \Omega .
$$

Secondly, we show that the operators $G_{\alpha}^{0}$ are bounded with norm $1 / \alpha$, for all $\alpha>0$. To do this, it suffices to show that

$$
G_{\alpha}^{0} 1(x) \leq \frac{1}{\alpha} \quad \text { on } \bar{\Omega}
$$


since the $G_{\alpha}^{0}$ are non-negative on $C(\bar{\Omega})$.

If we let

$$
u(x):=\alpha G_{\alpha}^{0} 1(x)-1 \in W^{2, p}(\Omega),
$$

then it follows that

$$
(A-\alpha) u(x)=-\alpha+(\alpha-c(x))=-c(x) \geq 0 \quad \text { in } \Omega,
$$

and that

$$
u=-1 \quad \text { on } \partial \Omega .
$$

Thus, by applying Theorem 4.4 with $A:=A-\alpha$ we obtain that

$$
\alpha G_{\alpha}^{0} 1(x)-1=u(x) \leq 0 \quad \text { on } \bar{\Omega} .
$$

This proves the desired assertion $\left(6.4^{\prime}\right)$.

Assertion (b): It suffices to note that the function $G_{\alpha}^{0} f \in W^{2, p}(\Omega) \cap W_{0}^{1, p}(\Omega)$ is the unique solution of the Dirichlet problem (6.2).

Assertion (c): This is an immediate consequence of the uniqueness theorem for problem (6.1) (Theorem 4.1). Indeed, it follows that the function

$$
u:=G_{\alpha}^{0} f-G_{\beta}^{0} f+(\alpha-\beta) G_{\alpha}^{0}\left(G_{\beta}^{0} f\right) \in W^{2, p}(\Omega)
$$

satisfies the equation

$$
\begin{aligned}
(\alpha-A) u & =f-(\alpha-A) G_{\beta}^{0} f+(\alpha-\beta) G_{\beta}^{0} f \\
& =f-(\beta-A+\alpha-\beta) G_{\beta}^{0} f+(\alpha-\beta) G_{\beta}^{0} f \\
& =f-f-(\alpha-\beta) G_{\beta}^{0} f+(\alpha-\beta) G_{\beta}^{0} f \\
& =0 \quad \text { in } \Omega,
\end{aligned}
$$

and the boundary condition

$$
u=0 \quad \text { on } \partial \Omega .
$$

By applying Theorem 4.1 to the operator $A-\alpha$, we obtain that

$$
u=0 \quad \text { in } \Omega .
$$

This proves the resolvent equation (6.5) for $f \in C(\bar{\Omega})$.

Assertion (d): First, let $f(x)$ be an arbitrary function in $C(\bar{\Omega})$ satisfying $f=0$ on $\partial \Omega$. Then it follows from the uniqueness theorem for problem (6.1) that we have, for all $\alpha, \beta$,

$$
f-\alpha G_{\alpha}^{0} f=G_{\alpha}^{0}((\beta-A) f)-\beta G_{\alpha}^{0} f .
$$

Thus we have, by estimate (6.4),

$$
\left\|f-\alpha G_{\alpha}^{0} f\right\|_{C(\bar{\Omega})} \leq \frac{1}{\alpha}\|(\beta-A) f\|_{C(\bar{\Omega})}+\frac{\beta}{\alpha}\|f\|_{C(\bar{\Omega})},
$$

so that

$$
\lim _{\alpha \rightarrow+\infty}\left\|f-\alpha G_{\alpha}^{0} f\right\|_{C(\bar{\Omega})}=0 .
$$


To prove assertion (6.6), let $f(x)$ be an arbitrary function in $C(\bar{\Omega})$ and $x_{0}$ an arbitrary point of $\Omega$. Take a function $\psi(x) \in C(\bar{\Omega})$ such that

$$
\begin{cases}0 \leq \psi(x) \leq 1 & \text { on } \bar{\Omega} \\ \psi(x)=0 & \text { in a neighborhood of } x_{0} \\ \psi(x)=1 & \text { near the boundary } \partial \Omega\end{cases}
$$

Then it follows from the non-negativity of $G_{\alpha}^{0}$ and estimate (6.4) that

$$
0 \leq \alpha G_{\alpha}^{0} \psi\left(x_{0}\right)+\alpha G_{\alpha}^{0}(1-\psi)\left(x_{0}\right)=\alpha G_{\alpha}^{0} 1\left(x_{0}\right) \leq 1 .
$$

However, by applying assertion (6.7) to the function $1-\psi(x)$ we obtain that

$$
\lim _{\alpha \rightarrow+\infty} \alpha G_{\alpha}^{0}(1-\psi)\left(x_{0}\right)=(1-\psi)\left(x_{0}\right)=1 .
$$

In view of inequalities (6.9), this implies that

$$
\lim _{\alpha \rightarrow+\infty} \alpha G_{\alpha}^{0} \psi\left(x_{0}\right)=0 .
$$

Thus, since we have

$$
-\|f\|_{C(\bar{\Omega})} \psi \leq f \psi \leq\|f\|_{C(\bar{\Omega})} \psi \quad \text { on } \bar{\Omega},
$$

it follows that

$$
\left|\alpha G_{\alpha}^{0}(f \psi)\left(x_{0}\right)\right| \leq\|f\|_{C(\bar{\Omega})} \alpha G_{\alpha}^{0} \psi\left(x_{0}\right) \rightarrow 0 \quad \text { as } \alpha \rightarrow+\infty .
$$

Therefore, by applying assertion (6.7) to the function $(1-\psi(x)) f(x)$ we obtain that

$$
f\left(x_{0}\right)=((1-\psi) f)\left(x_{0}\right)=\lim _{\alpha \rightarrow+\infty} \alpha G_{\alpha}^{0}((1-\psi) f)\left(x_{0}\right)=\lim _{\alpha \rightarrow+\infty} \alpha G_{\alpha}^{0} f\left(x_{0}\right) .
$$

(ii) Assertion (e): First, let $\varphi\left(x^{\prime}\right)$ be an arbitrary function in $C^{2}(\partial \Omega)$ such that $\varphi \geq 0$ on $\partial \Omega$. Then we have

$$
\begin{cases}(A-\alpha)\left(-H_{\alpha} \varphi\right)=0 & \text { in } \Omega \\ -H_{\alpha} \varphi=-\varphi \leq 0 & \text { on } \partial \Omega\end{cases}
$$

Therefore, byapplying Theorem 4.4 (the weak maximum principle) with $A:=$ $A-\alpha$ to the function $u:=-H_{\alpha} \varphi$ we obtain that

$$
H_{\alpha} \varphi \geq 0 \text { on } \bar{\Omega} \text {. }
$$

This proves the non-negativity of $H_{\alpha}$.

In order to prove the boundedness of $H_{\alpha}$

$$
\left\|H_{\alpha}\right\|=1,
$$

it suffices to show that

$$
H_{\alpha} 1(x) \leq 1 \quad \text { in } \Omega
$$

since $H_{\alpha}$ is non-negative. 
To do this, we remark that the function $H_{\alpha} 1-1$ satisfies the conditions

$$
\begin{cases}(A-\alpha)\left(H_{\alpha} 1-1\right)=-c(x)+\alpha \geq 0 & \text { in } \Omega, \\ H_{\alpha} 1-1=0 & \text { on } \partial \Omega .\end{cases}
$$

Therefore, by applying Theorem 4.4 with $A:=A-\alpha$ and $u:=H_{\alpha} 1-1$ we obtain that

$$
H_{\alpha} 1(x)-1 \leq 0 \quad \text { in } \Omega .
$$

Since the space $C^{2}(\partial \Omega)$ is dense in $C(\partial \Omega)$, it follows that the operator $H_{\alpha}: C^{2}(\partial \Omega) \rightarrow C(\bar{\Omega})$ can be uniquely extended to a non-negative, bounded linear operator, denoted again by $H_{\alpha}: C(\partial \Omega) \rightarrow C(\bar{\Omega})$.

Assertion (f): This follows from formula (6.3), since the space $C^{2}(\partial \Omega)$ is dense in $C(\partial \Omega)$ and since the operator $H_{\alpha}: C(\partial \Omega) \rightarrow C(\bar{\Omega})$ is bounded.

Assertion $(g)$ : We find from the uniqueness theorem for problem (6.3) (Theorem 4.1) that formula (6.8) holds for all $\varphi \in C^{2}(\partial \Omega)$. Hence it holds true for all $\varphi \in C(\partial \Omega)$, since the space $C^{2}(\partial \Omega)$ is dense in $C(\partial \Omega)$ and since the operators $G_{\alpha}^{0}$ and $H_{\alpha}$ are bounded.

The proof of Theorem 6.1 is now complete.

Summing up, we have the following diagrams for the operators $G_{\alpha}^{0}: C(\bar{\Omega}) \rightarrow$ $C(\bar{\Omega})$ and $H_{\alpha}: C(\partial \Omega) \rightarrow C(\bar{\Omega}):$

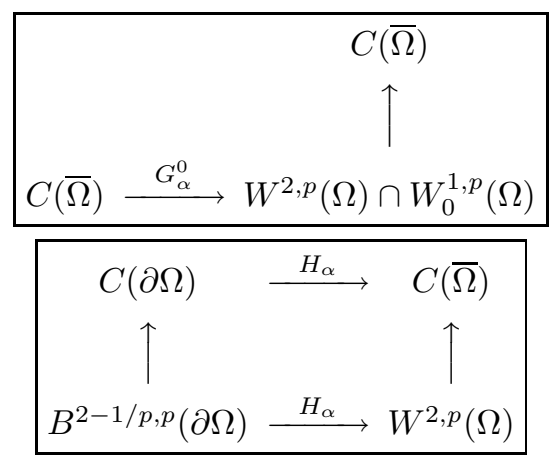

\subsection{General Boundary Value Problems}

Let $L$ be a first-order, Ventcel' boundary condition of the form (1.2)

$$
L u:=\mu\left(x^{\prime}\right) \frac{\partial u}{\partial \mathbf{n}}+\beta\left(x^{\prime}\right) \cdot u+\gamma\left(x^{\prime}\right) u-\delta\left(x^{\prime}\right)\left(\left.A u\right|_{\partial \Omega}\right) \quad \text { on } \partial \Omega .
$$

In this subsection we consider the following general boundary value problem in the framework of the spaces of continuous functions:

$$
\begin{cases}(\alpha-A) u=f & \text { in } \Omega, \\ L u=0 & \text { on } \partial \Omega .\end{cases}
$$

To do this, we introduce three linear operators associated with problem (6.10).

(I) First, we introduce a linear operator

$$
\overline{\mathcal{A}}: C(\bar{\Omega}) \longrightarrow C(\bar{\Omega})
$$

as follows. 
(a) The domain $D(\overline{\mathcal{A}})$ of $\overline{\mathcal{A}}$ is the space

$$
D(\overline{\mathcal{A}})=\left\{u \in W^{2, p}(\Omega): A u \in C(\bar{\Omega})\right\} .
$$

(b) $\overline{\mathcal{A}} u=A u, u \in D(\overline{\mathcal{A}})$.

Then we have the following:

Lemma 6.2. The operator $\overline{\mathcal{A}}$ is a densely defined, closed linear operator in the space $C(\bar{\Omega})$.

Proof. First, by the definition of $\overline{\mathcal{A}}$ and $\mathfrak{A}_{N}$ it follows that

$$
\mathfrak{A}_{N} \subset \overline{\mathcal{A}} \text {. }
$$

This proves the density of the domain $D(\overline{\mathcal{A}})$ in $C(\bar{\Omega})$, since the domain $D\left(\mathfrak{A}_{N}\right)$ is dense in $C(\bar{\Omega})$ (see assertion (5.6)).

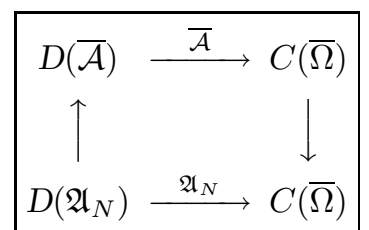

Now, let $(u, v)$ be an arbitrary element of the product space $C(\bar{\Omega}) \oplus C(\bar{\Omega})$ such that there exists a sequence $\left\{u_{n}\right\} \subset \overline{\mathcal{A}}$ which satisfies the conditions

$$
\begin{aligned}
& u_{n} \longrightarrow u \quad \text { in } C(\bar{\Omega}), \\
& A u_{n} \longrightarrow v \quad \text { in } C(\bar{\Omega}) .
\end{aligned}
$$

Then we have, by the boundedness of $G_{\alpha}^{0}$,

$$
G_{\alpha}^{0}\left(A u_{n}\right)=\alpha G_{\alpha}^{0} u_{n}-u_{n} \longrightarrow \alpha G_{\alpha}^{0} u-u \quad \text { in } C(\bar{\Omega}),
$$

and also

$$
G_{\alpha}^{0}\left(A u_{n}\right) \longrightarrow G_{\alpha}^{0} v \quad \text { in } C(\bar{\Omega}) .
$$

This proves that

$$
u=\alpha G_{\alpha}^{0} u-G_{\alpha}^{0} v \in W^{2, p}(\Omega) .
$$

Thus, by applying the operator $\alpha-A$ to the both hand sides of formula (6.11) we obtain that

$$
(\alpha-A) u=\alpha(\alpha-A) G_{\alpha}^{0} u-(\alpha-A) G_{\alpha}^{0} v=\alpha u-v,
$$

so that

$$
A u=v \in C(\bar{\Omega}) .
$$

Summing up, we have proved that

$$
\begin{aligned}
& u \in D(\overline{\mathcal{A}}), \\
& \overline{\mathcal{A}} u=v .
\end{aligned}
$$

This proves the closedness of $\overline{\mathcal{A}}$.

The proof of Lemma 6.2 is complete. 
Remark 6.1. The domain $D(\overline{\mathcal{A}})$ does not depend on $p$, for $N<p<\infty$ (see Section 6.4).

The extended operators $G_{\alpha}^{0}: C(\bar{\Omega}) \rightarrow C(\bar{\Omega})$ and $H_{\alpha}: C(\partial \Omega) \rightarrow C(\bar{\Omega}), \alpha>0$, still satisfy formulas (6.2) and (6.3) respectively in the following sense:

Lemma 6.3. (i) For any $f \in C(\bar{\Omega})$, we have the assertion

$$
\left\{\begin{array}{l}
G_{\alpha}^{0} f \in D(\overline{\mathcal{A}}), \\
(\alpha I-\overline{\mathcal{A}}) G_{\alpha}^{0} f=f .
\end{array}\right.
$$

(ii) For any $\varphi \in C(\partial \Omega)$, we have the assertion

$$
\left\{\begin{array}{l}
H_{\alpha} \varphi \in D(\overline{\mathcal{A}}), \\
(\alpha I-\overline{\mathcal{A}}) H_{\alpha} \varphi=0 .
\end{array}\right.
$$

Proof. Assertion (i): If $f \in C(\bar{\Omega})$, then it follows from the definition of $G_{\alpha}^{0}$ that

$$
\begin{aligned}
& G_{\alpha}^{0} f \in W^{2, p}(\Omega), \\
& A\left(G_{\alpha}^{0} f\right)=\alpha G_{\alpha}^{0} f-f \in C(\bar{\Omega}) .
\end{aligned}
$$

This implies assertion (6.12).

Assertion (ii): If $\varphi \in C(\partial \Omega)$, we can find a sequence $\left\{\varphi_{j}\right\}$ in the space $C^{2}(\partial \Omega)$ such that

$$
\varphi_{j} \longrightarrow \varphi \text { in } C(\partial \Omega) .
$$

Hence, we have, by the boundedness of $H_{\alpha}$,

$$
H_{\alpha} \varphi_{j} \longrightarrow H_{\alpha} \varphi \text { in } C(\bar{\Omega})
$$

However, it follows that

$$
\begin{aligned}
& H_{\alpha} \varphi_{j} \in W^{2, p}(\Omega), \\
& A\left(H_{\alpha} \varphi_{j}\right)=\alpha H_{\alpha} \varphi_{j} \in C(\bar{\Omega}),
\end{aligned}
$$

so that

$$
H_{\alpha} \varphi_{j} \in D(\overline{\mathcal{A}})
$$

Therefore, we have proved that

$$
\begin{aligned}
& H_{\alpha} \varphi_{j} \in D(\overline{\mathcal{A}}), \\
& H_{\alpha} \varphi_{j} \longrightarrow H_{\alpha} \varphi \quad \text { in } C(\bar{\Omega}), \\
& A\left(H_{\alpha} \varphi_{j}\right) \longrightarrow \alpha H_{\alpha} \varphi \quad \text { in } C(\bar{\Omega}) .
\end{aligned}
$$

This proves assertion (6.13), since the operator $\overline{\mathcal{A}}$ is closed.

The proof of Lemma 6.3 is complete.

Corollary 6.4. Every function $u$ in $D(\overline{\mathcal{A}})$ can be written in the form

$$
u=G_{\alpha}^{0}((\alpha I-\overline{\mathcal{A}}) u)+H_{\alpha}\left(\left.u\right|_{\partial \Omega}\right), \quad \alpha>0 .
$$


Proof. We let

$$
w=u-G_{\alpha}^{0}((\alpha I-\overline{\mathcal{A}}) u)-H_{\alpha}\left(\left.u\right|_{\partial \Omega}\right) .
$$

Then it follows from Lemma 6.3 that the function $w$ is in $D(\overline{\mathcal{A}})$ and satisfies the conditions

$$
\begin{cases}(\alpha I-\overline{\mathcal{A}}) w=0 & \text { in } \Omega, \\ w=0 & \text { on } \partial \Omega .\end{cases}
$$

Therefore, we can apply Theorem 4.1 to the operator $A-\alpha$ to obtain that

$$
w=0 .
$$

This proves formula (6.14).

The proof of Corollary 6.4 is complete.

(II) Secondly, we introduce a linear operator

$$
\overline{L G_{\alpha}^{0}}: C(\bar{\Omega}) \longrightarrow C(\partial \Omega)
$$

as follows.

(a) The domain $D\left(\overline{L G_{\alpha}^{0}}\right)$ of $\overline{L G_{\alpha}^{0}}$ is the space $C(\bar{\Omega})$.

(b) $\overline{L G_{\alpha}^{0}} f=L\left(G_{\alpha}^{0} f\right)=\mu\left(x^{\prime}\right) \frac{\partial}{\partial \mathbf{n}}\left(G_{\alpha}^{0} f\right)+\delta\left(x^{\prime}\right)\left(\left.f\right|_{\partial \Omega}\right), f \in D\left(\overline{L G_{\alpha}^{0}}\right)$.

The operator $\overline{L G_{\alpha}^{0}}: C(\bar{\Omega}) \rightarrow C(\partial \Omega)$ can be visualized as follows:

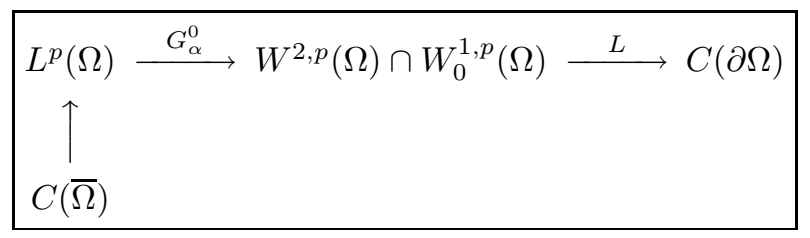

Here it should be emphasized that we have, by Sobolev's imbedding theorem (see [26, Theorem 4.12, Part II]),

$$
G_{\alpha}^{0} f \in W^{2, p}(\Omega) \subset C^{1}(\bar{\Omega}),
$$

since $2-N / p>1$ for $N<p<\infty$.

Then we have the following:

Lemma 6.5. The operators $\overline{L G_{\alpha}^{0}}: C(\bar{\Omega}) \rightarrow C(\partial \Omega)$ are non-negative and bounded for all $\alpha>0$.

Proof. Let $f$ be an arbitrary function in $D\left(\overline{L G_{\alpha}^{0}}\right)$ such that $f(x) \geq 0$ on $\bar{\Omega}$. Then we have the assertions

$$
\begin{cases}G_{\alpha}^{0} f \in C^{1}(\bar{\Omega}), & \\ G_{\alpha}^{0} f \geq 0 & \text { on } \bar{\Omega}, \\ G_{\alpha}^{0} f=0 & \text { on } \partial \Omega,\end{cases}
$$

and so

$$
\overline{L G_{\alpha}^{0}} f\left(x^{\prime}\right)=L\left(G_{\alpha}^{0} f\right)\left(x^{\prime}\right)=\mu\left(x^{\prime}\right) \frac{\partial}{\partial \mathbf{n}}\left(G_{\alpha}^{0} f\right)\left(x^{\prime}\right)+\delta\left(x^{\prime}\right) f\left(x^{\prime}\right) \geq 0 \quad \text { on } \partial \Omega .
$$


This proves that the operator $\overline{L G_{\alpha}^{0}}$ is non-negative.

By the non-negativity of $\overline{L G_{\alpha}^{0}}$, we have, for all $f \in D\left(\overline{L G_{\alpha}^{0}}\right)$,

$$
-\overline{L G_{\alpha}^{0}}\|f\|_{C(\bar{\Omega})} \leq \overline{L G_{\alpha}^{0}} f \leq \overline{L G_{\alpha}^{0}}\|f\|_{C(\bar{\Omega})} \quad \text { on } \partial \Omega .
$$

This implies the boundedness of $\overline{L G_{\alpha}^{0}}$ with norm

$$
\left\|\overline{L G_{\alpha}^{0}}\right\|=\left\|L\left(G_{\alpha}^{0} 1\right)\right\|_{C(\partial \Omega)} .
$$

The proof of Lemma 6.5 is complete.

Remark 6.2. Similarly, we can prove that the operators

$$
L G_{\alpha}^{0}: L^{\infty}(\Omega) \longrightarrow C(\partial \Omega)
$$

are non-negative and bounded for all $\alpha>0$, with norm

$$
\left\|L G_{\alpha}^{0}\right\|=\left\|L\left(G_{\alpha}^{0} 1\right)\right\|_{C(\partial \Omega)} .
$$

This can be visualized as follows:

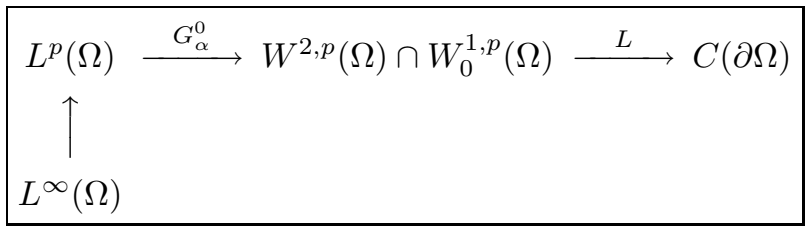

The next lemma states a fundamental relationship between the operators $\overline{L G_{\alpha}^{0}}$ and $\overline{L G_{\beta}^{0}}$ for $\alpha, \beta>0$ :

Lemma 6.6. For any $\alpha, \beta>0$, we have the equation

$$
\overline{L G_{\alpha}^{0}} f-\overline{L G_{\beta}^{0}} f+(\alpha-\beta) \overline{L G_{\alpha}^{0}}\left(G_{\beta}^{0} f\right)=0, \quad f \in C(\bar{\Omega}) .
$$

Proof. We have, by the resolvent equation (6.5),

$$
G_{\alpha}^{0} f-G_{\beta}^{0} f+(\alpha-\beta) G_{\alpha}^{0}\left(G_{\beta}^{0} f\right)=0 .
$$

Therefore, formula (6.15) follows by applying the operator $L$ to the both hand sides of equation (6.5).

The proof of Lemma 6.6 is complete.

Remark 6.3. The equation (6.15) remains valid for $f \in L^{\infty}(\Omega)$ :

$$
L G_{\alpha}^{0} f-L G_{\beta}^{0} f+(\alpha-\beta) \overline{L G_{\alpha}^{0}}\left(G_{\beta}^{0} f\right)=0, \quad f \in L^{\infty}(\Omega) .
$$

Indeed, it suffices to note that the function

$$
u:=G_{\alpha}^{0} f-G_{\beta}^{0} f+(\alpha-\beta) G_{\alpha}^{0}\left(G_{\beta}^{0} f\right) \in W^{2, p}(\Omega)
$$

is a unique solution of the Dirichlet problem

$$
\begin{cases}(\alpha-A) u=0 & \text { almost everywhere in } \Omega, \\ u=0 & \text { on } \partial \Omega\end{cases}
$$


(III) Finally, we introduce a linear operator

$$
L H_{\alpha}: C(\partial \Omega) \longrightarrow C(\partial \Omega)
$$

as follows.

(a) The domain $D\left(L H_{\alpha}\right)$ of $L H_{\alpha}$ is the space $B^{2-1 / p, p}(\partial \Omega)$.

(b) $L H_{\alpha} \psi=L\left(H_{\alpha} \psi\right)=\mu\left(x^{\prime}\right) \frac{\partial}{\partial \mathbf{n}}\left(H_{\alpha} \psi\right)+\beta\left(x^{\prime}\right) \cdot \psi+\gamma\left(x^{\prime}\right) \psi-\alpha \delta\left(x^{\prime}\right) \psi, \psi \in$ $D\left(L H_{\alpha}\right)$.

The operator $L H_{\alpha}: C(\partial \Omega) \rightarrow C(\partial \Omega)$ can be visualized as follows:

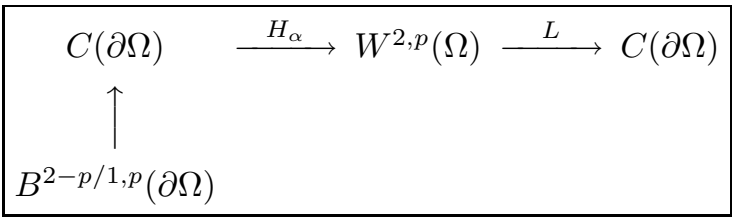

Then we have the following:

Lemma 6.7. The operator $L H_{\alpha}, \alpha>0$, has its minimal closed extension $\overline{L H_{\alpha}}$ in the space $C(\partial \Omega)$.

Proof. We apply part (i) of Theorem 3.2 with $K:=\partial \Omega$ to the operator $B:=$ $L H_{\alpha}$. To do this, it suffices to show that the operator $L H_{\alpha}$ satisfies condition $\left(\beta^{\prime}\right)$ with $K:=\partial \Omega$ (or condition $(\beta)$ with $K:=K_{0}=\partial \Omega$ ) of the same theorem.

Assume that a function $\varphi$ in the domain $D\left(L H_{\alpha}\right)=B^{2-1 / p, p}(\partial \Omega)$ takes its positive maximum at some point $x_{0}^{\prime}$ of $\partial \Omega$. Since the function $H_{\alpha} \varphi$ is in $W^{2, p}(\Omega)$ and satisfies the conditions

$$
\begin{cases}(A-\alpha) H_{\alpha} \varphi=0 & \text { in } \Omega, \\ H_{\alpha} \varphi=\varphi & \text { on } \partial \Omega,\end{cases}
$$

by applying the weak maximum principle (Theorem 4.4) with $A:=A-\alpha$ to the function $H_{\alpha} \psi$, we find that the function $H_{\alpha} \varphi$ takes its positive maximum at a boundary point $x_{0}^{\prime} \in \partial \Omega$. Thus we can apply the Hopf boundary point lemma (Lemma A.2) to obtain that

$$
\frac{\partial}{\partial \mathbf{n}}\left(H_{\alpha} \varphi\right)\left(x_{0}^{\prime}\right)<0 .
$$

However, it should be noticed that the coefficients of the boundary condition $L$ satisfy the conditions

$$
\begin{array}{ll}
\mu\left(x^{\prime}\right)>0 & \text { on } \partial \Omega, \\
\gamma\left(x^{\prime}\right)<0 & \text { on } \partial \Omega, \\
\delta\left(x^{\prime}\right) \geq 0 & \text { on } \partial \Omega
\end{array}
$$

Hence we have

$$
L H_{\alpha} \varphi\left(x_{0}^{\prime}\right)=\mu\left(x_{0}^{\prime}\right) \frac{\partial}{\partial \mathbf{n}}\left(H_{\alpha} \varphi\right)\left(x_{0}^{\prime}\right)+\beta\left(x_{0}^{\prime}\right) \cdot \varphi\left(x_{0}^{\prime}\right)
$$




$$
\begin{aligned}
& +\gamma\left(x_{0}^{\prime}\right) \varphi\left(x_{0}^{\prime}\right)-\alpha \delta\left(x_{0}^{\prime}\right) \varphi\left(x_{0}^{\prime}\right) \\
= & \mu\left(x_{0}^{\prime}\right) \frac{\partial}{\partial \mathbf{n}}\left(H_{\alpha} \varphi\right)\left(x_{0}^{\prime}\right)+\gamma\left(x_{0}^{\prime}\right) \varphi\left(x_{0}^{\prime}\right)-\alpha \delta\left(x_{0}^{\prime}\right) \varphi\left(x_{0}^{\prime}\right) \\
< & 0 .
\end{aligned}
$$

This verifies condition $\left(\beta^{\prime}\right)$ of Theorem 3.2.

Therefore, Lemma 6.7 follows from an application of Theorem 3.2.

Remark 6.4. The operator $\overline{L H_{\alpha}}$ enjoys the following property:

If a function $\varphi$ in the domain $D\left(\overline{L H_{\alpha}}\right)$ takes its positive maximum at some point $x_{0}^{\prime}$ of $\partial \Omega$, then we have the inequality

$$
\overline{L H_{\alpha}} \varphi\left(x_{0}^{\prime}\right) \leq 0 .
$$

The next lemma states a fundamental relationship between the operators $\overline{L H_{\alpha}}$ and $\overline{L H_{\beta}}$ for $\alpha, \beta>0$ :

Lemma 6.8. The domain $D\left(\overline{L H_{\alpha}}\right)$ of $\overline{L H_{\alpha}}$ does not depend on $\alpha>0$; so we denote by $\mathcal{D}$ the common domain. Then we have, for all $\alpha, \beta>0$, the equation

$$
\overline{L H_{\alpha}} \varphi-\overline{L H_{\beta}} \varphi+(\alpha-\beta) \overline{L G_{\alpha}^{0}}\left(H_{\beta} \varphi\right)=0, \quad \varphi \in \mathcal{D} .
$$

Proof. Let $\varphi$ be an arbitrary function in $D\left(\overline{L H_{\beta}}\right)$, and choose a sequence $\left\{\varphi_{j}\right\}$ in $D\left(L H_{\beta}\right)=B^{2-1 / p, p}(\partial \Omega)$ such that

$$
\begin{cases}\varphi_{j} \longrightarrow \varphi & \text { in } C(\partial \Omega), \\ L H_{\beta} \varphi_{j} \longrightarrow \overline{L H_{\beta}} \varphi & \text { in } C(\partial \Omega) .\end{cases}
$$

Then it follows from the boundedness of $H_{\beta}$ and $\overline{L G_{\alpha}^{0}}$ that

$$
\overline{L G_{\alpha}^{0}}\left(H_{\beta} \varphi_{j}\right) \longrightarrow \overline{L G_{\alpha}^{0}}\left(H_{\beta} \varphi\right) \quad \text { in } C(\partial \Omega) .
$$

Therefore, by using formula (6.8) with $\varphi:=\varphi_{j}$ we obtain that

$$
\begin{aligned}
L H_{\alpha} \varphi_{j} & =L H_{\beta} \varphi_{j}-(\alpha-\beta) \overline{L G_{\alpha}^{0}}\left(H_{\beta} \varphi_{j}\right) \\
& \longrightarrow \overline{L H_{\beta}} \varphi-(\alpha-\beta) \overline{L G_{\alpha}^{0}}\left(H_{\beta} \varphi\right) \quad \text { in } C(\partial \Omega) .
\end{aligned}
$$

Since the operator $\overline{L H_{\alpha}}$ is closed, it follows that

$$
\left\{\begin{array}{l}
\varphi \in D\left(\overline{L H_{\alpha}}\right), \\
\overline{L H_{\alpha}} \varphi=\overline{L H_{\beta}} \varphi-(\alpha-\beta) \overline{L G_{\alpha}^{0}}\left(H_{\beta} \varphi\right) .
\end{array}\right.
$$

This proves the desired equation (6.18).

Conversely, we have, by interchanging $\alpha$ and $\beta$,

$$
D\left(\overline{L H_{\alpha}}\right) \subset D\left(\overline{L H_{\beta}}\right),
$$

and so

$$
D\left(\overline{L H_{\alpha}}\right)=D\left(\overline{L H_{\beta}}\right) .
$$

The proof of Lemma 6.8 is complete. 


\subsection{General Existence Theorem for Feller Semigroups}

Now we can give a general existence theorem for Feller semigroups on $\partial \Omega$ in terms of boundary value problem (6.10). The next theorem asserts that the operator $\overline{L H_{\alpha}}$ is the infinitesimal generator of some Feller semigroup on $\partial \Omega$ if and only if problem (6.10) is solvable for sufficiently many functions $\varphi$ in the space $C(\partial \Omega)$ :

Theorem 6.9. (i) If the operator $\overline{L H_{\alpha}}, \alpha>0$, is the infinitesimal generator of a Feller semigroup on $\partial \Omega$, then, for each constant $\lambda>0$, the boundary value problem

$$
\begin{cases}(\alpha-A) u=0 & \text { in } \Omega, \\ (\lambda-L) u=\varphi & \text { on } \partial \Omega\end{cases}
$$

has a solution $u \in W^{2, p}(\Omega)$ for any $\varphi$ in some dense subset of $C(\partial \Omega)$.

(ii) Conversely, if, for some constant $\lambda \geq 0$, problem (6.19) has a solution $u \in W^{2, p}(\Omega)$ for any $\varphi$ in some dense subset of $C(\partial \Omega)$, then the operator $\overline{L H_{\alpha}}$ is the infinitesimal generator of some Feller semigroup on $\partial \Omega$.

Proof. Assertion (i): If the operator $\overline{L H_{\alpha}}$ generates a Feller semigroup on $\partial \Omega$, by applying part (i) of Theorem 3.2 with $K:=\partial \Omega$ to the operator $\overline{L H_{\alpha}}$ we obtain that

$$
R\left(\lambda I-\overline{L H_{\alpha}}\right)=C(\partial \Omega) \text { for each } \lambda>0 .
$$

This implies that the range $R\left(\lambda I-L H_{\alpha}\right)$ is a dense subset of $C(\partial \Omega)$ for each $\lambda>0$. However, if $\varphi \in C(\partial \Omega)$ is in the range $R\left(\lambda I-L H_{\alpha}\right)$, and if $\varphi=$ $\left(\lambda I-L H_{\alpha}\right) \psi$ with $\psi \in B^{2-1 / p, p}(\partial \Omega)$, then the function $u=H_{\alpha} \psi \in W^{2, p}(\Omega)$ is a solution of problem (6.19). This proves assertion (i).

Assertion (ii): We apply part (ii) of Theorem 3.2 with $K:=\partial \Omega$ to the operator $L H_{\alpha}$. To do this, it suffices to show that the operator $L H_{\alpha}$ satisfies condition $(\gamma)$ of the same theorem, since it satisfies condition $\left(\beta^{\prime}\right)$, as is shown in the proof of Lemma 6.7 .

By the uniqueness theorem for problem (6.1) (Theorem 4.1), it follows that every function $u \in W^{2, p}(\Omega)$ which satisfies the equation

$$
(\alpha-A) u=0 \quad \text { in } \Omega
$$

can be written in the form

$$
u=H_{\alpha}\left(\left.u\right|_{\partial \Omega}\right),\left.\quad u\right|_{\partial \Omega} \in B^{2-1 / p, p}(\partial \Omega)=D\left(L H_{\alpha}\right) .
$$

Thus we find that if there exists a solution $u \in W^{2, p}(\Omega)$ of problem (6.19) for a function $\varphi \in C(\partial \Omega)$, then we have

$$
\left(\lambda I-L H_{\alpha}\right)\left(\left.u\right|_{\partial \Omega}\right)=\varphi
$$

and so

$$
\varphi \in R\left(\lambda I-L H_{\alpha}\right) .
$$

Therefore, if there exists a constant $\lambda \geq 0$ such that problem (6.19) has a solution $u$ in $W^{2, p}(\Omega)$ for any $\varphi$ in some dense subset of $C(\partial \Omega)$, then the range $R\left(\lambda I-L H_{\alpha}\right)$ is dense in $C(\partial \Omega)$. This verifies condition $(\gamma)$ (with $\alpha_{0}:=\lambda$ ) of Theorem 3.2. Hence assertion (ii) follows from an application of the same theorem.

The proof of Theorem 6.9 is complete. 
We conclude this subsection by giving a precise meaning to the boundary conditions $L u$ for functions $u$ in the domain $D(\overline{\mathcal{A}})$.

We let

$$
\begin{aligned}
D(L) & =\left\{u \in D(\overline{\mathcal{A}}):\left.u\right|_{\partial \Omega} \in \mathcal{D}\right\} \\
& =\left\{u \in W^{2, p}(\Omega): A u \in C(\bar{\Omega}),\left.u\right|_{\partial \Omega} \in \mathcal{D}\right\},
\end{aligned}
$$

where $\mathcal{D}$ is the common domain of the operators $\overline{L H_{\alpha}}, \alpha>0$ (see Lemma 6.8). It should be noticed that the domain $D(L)$ contains $W^{2, p}(\Omega)$, since $B^{2-1 / p, p}(\partial \Omega)=$ $D\left(L H_{\alpha}\right) \subset \mathcal{D}$. Moreover, Corollary 6.4 asserts that every function $u$ in $D(L) \subset$ $D(\overline{\mathcal{A}})$ can be written in the form $(6.14)$

$$
u=G_{\alpha}^{0}((\alpha I-\overline{\mathcal{A}}) u)+H_{\alpha}\left(\left.u\right|_{\partial \Omega}\right), \quad \alpha>0 .
$$

Then we define the boundary condition $L u$ by the formula

$$
L u=\overline{L G_{\alpha}^{0}}((\alpha I-\overline{\mathcal{A}}) u)+\overline{L H_{\alpha}}\left(\left.u\right|_{\partial \Omega}\right) .
$$

The next lemma justifies the definition (6.20) of $L u$ for $u \in D(L)$ :

Lemma 6.10. The right-hand side of formula (6.20) depends only on $u$, not on the choice of expression (6.14).

Proof. Assume that

$$
\begin{aligned}
u & =G_{\alpha}^{0}((\alpha I-\overline{\mathcal{A}}) u)+H_{\alpha}\left(\left.u\right|_{\partial \Omega}\right) \\
& =G_{\beta}^{0}((\beta I-\overline{\mathcal{A}}) u)+H_{\beta}\left(\left.u\right|_{\partial \Omega}\right),
\end{aligned}
$$

where $\alpha>0, \beta>0$. Then it follows from formula (6.15) with $f:=(\alpha I-\overline{\mathcal{A}}) u$ and formula $(6.18)$ with $\psi:=\left.u\right|_{\partial \Omega}$ that

$$
\begin{aligned}
& \overline{L G_{\alpha}^{0}}((\alpha I-\overline{\mathcal{A}}) u)+\overline{L H_{\alpha}}\left(\left.u\right|_{\partial \Omega}\right) \\
= & \overline{L G_{\beta}^{0}}((\alpha I-\overline{\mathcal{A}}) u)-(\alpha-\beta) \overline{L G_{\alpha}^{0}} G_{\beta}^{0}((\alpha I-\overline{\mathcal{A}}) u) \\
& +\overline{L H_{\beta}}\left(\left.u\right|_{\partial \Omega}\right)-(\alpha-\beta) \overline{L G_{\alpha}^{0}} H_{\beta}\left(\left.u\right|_{\partial \Omega}\right) \\
= & \overline{L G_{\beta}^{0}}((\beta I-A) u)+\overline{L H_{\beta}}\left(\left.u\right|_{\partial \Omega}\right) \\
& +(\alpha-\beta)\left\{\overline{L G_{\beta}^{0}} u-\overline{L G_{\alpha}^{0}} G_{\beta}^{0}(\alpha I-\overline{\mathcal{A}}) u-\overline{L G_{\alpha}^{0}} H_{\beta}\left(\left.u\right|_{\partial \Omega}\right)\right\} .
\end{aligned}
$$

However, the last term of formula (6.21) vanishes. Indeed, it follows from formula (6.15) with $f:=u$ that

$$
\begin{aligned}
& \overline{L G_{\beta}^{0}} u-\overline{L G_{\alpha}^{0}}\left(G_{\beta}^{0}(\alpha I-\overline{\mathcal{A}}) u\right)-\overline{L G_{\alpha}^{0}} H_{\beta}\left(\left.u\right|_{\partial \Omega}\right) \\
= & \overline{L G_{\beta}^{0}} u-\overline{L G_{\alpha}^{0}}\left(G_{\beta}^{0}(\beta I-\overline{\mathcal{A}}) u+H_{\beta}\left(\left.u\right|_{\partial \Omega}\right)+(\alpha-\beta) G_{\beta}^{0} u\right) \\
= & \overline{L G_{\beta}^{0}} u-\overline{L G_{\alpha}^{0}} u-(\alpha-\beta) \overline{L G_{\alpha}^{0}} G_{\beta}^{0} u \\
= & 0 .
\end{aligned}
$$

Therefore, we obtain from formula (6.21) that

$$
\overline{L G_{\alpha}^{0}}((\alpha I-\overline{\mathcal{A}}) u)+\overline{L H_{\alpha}}\left(\left.u\right|_{\partial \Omega}\right)=\overline{L G_{\beta}^{0}}((\beta I-\overline{\mathcal{A}}) u)+\overline{L H_{\beta}}\left(\left.u\right|_{\partial \Omega}\right) .
$$

This proves Lemma 6.10 . 


\subsection{Proof of Remark 6.1}

Finally, we prove that the domain

$$
D(\overline{\mathcal{A}})=\left\{u \in W^{2, p}(\Omega): A u \in C(\bar{\Omega})\right\}
$$

is independent of $N<p<\infty$.

We let

$$
\mathcal{D}_{p}:=\left\{u \in W^{2, p}(\Omega): A u \in C(\bar{\Omega})\right\} .
$$

In order to prove Remark 6.1 , it suffices to show that

$$
\mathcal{D}_{p_{1}}=\mathcal{D}_{p_{2}} \quad \text { for } N<p_{1}<p_{2}<\infty .
$$

First, it follows that

$$
\mathcal{D}_{p_{2}} \subset \mathcal{D}_{p_{1}},
$$

since we have $L^{p_{2}}(\Omega) \subset L^{p_{1}}(\Omega)$ for $p_{2}>p_{1}$.

Conversely, let $v$ be an arbitrary element of $\mathcal{D}_{p_{1}}$ :

$$
v \in W^{2, p_{1}}(\Omega), \quad A v \in C(\bar{\Omega}), L u=0 \text { on } \partial \Omega .
$$

Then, since we have $v, A v \in C(\bar{\Omega}) \subset L^{p_{2}}(\Omega)$, it follows from an application of Theorem 4.1 with $p:=p_{2}$ that

$$
G_{\alpha}^{0}((\alpha-A) v) \in W^{2, p_{2}}(\Omega) .
$$

Moreover, we can find a sequence $\left\{\varphi_{j}\right\}$ in $C^{2}(\partial \Omega)$ such that

$$
\left.\varphi_{j} \longrightarrow v\right|_{\partial \Omega} \text { in } C(\partial \Omega) \text {. }
$$

Then we have

$$
\begin{aligned}
& H_{\alpha} \varphi_{j} \in W^{2, p_{2}}(\Omega), \\
& H_{\alpha} \varphi_{j} \longrightarrow H_{\alpha}\left(\left.v\right|_{\partial \Omega}\right) \quad \text { in } C(\bar{\Omega}), \\
& A\left(H_{\alpha} \varphi_{j}\right)=\alpha H_{\alpha} \varphi_{j} \longrightarrow \alpha H_{\alpha}\left(\left.v\right|_{\partial \Omega}\right) \quad \text { in } C(\bar{\Omega}) .
\end{aligned}
$$

However, since $\overline{\mathcal{A}}: \mathcal{D}_{p_{2}} \rightarrow C(\bar{\Omega})$ is closed, it follows that

$$
H_{\alpha}\left(\left.v\right|_{\partial \Omega}\right) \in \mathcal{D}_{p_{2}} \subset W^{2, p_{2}}(\Omega) .
$$

Therefore, by applying Corollary 6.4 we obtain that

$$
v=G_{\alpha}^{0}((\alpha-A) v)+H_{\alpha}\left(\left.v\right|_{\partial \Omega}\right) \in W^{2, p_{2}}(\Omega) .
$$

This implies that $v \in \mathcal{D}_{p_{2}}$.

The proof of Remark 6.1 is complete.

\section{Proof of Theorem 1.1}

This section 7 is devoted to the proof of Theorem 1.1. The crucial point in the proof is that we consider the term $\delta\left(x^{\prime}\right)\left(\left.A u\right|_{\partial \Omega}\right)$ of sticking phenomenon in the Ventcel' boundary condition (1.2)

$$
L u=L_{0} u-\delta\left(x^{\prime}\right)\left(\left.A u\right|_{\partial \Omega}\right) \quad \text { on } \partial \Omega
$$


as a term of perturbation of the oblique derivative boundary condition (5.1)

$$
L_{0} u:=\mu\left(x^{\prime}\right) \frac{\partial u}{\partial \mathbf{n}}+\beta\left(x^{\prime}\right) \cdot u+\gamma\left(x^{\prime}\right) u \quad \text { on } \partial \Omega .
$$

More precisely, we make use of a generation theorem for Feller semigroups with oblique derivative boundary condition $L_{0}$ to verify all the conditions of a version of the Hille-Yosida theorem adapted to the present context (Theorem 3.2) for the operator $\mathfrak{A}$ defined by formula (1.3).

We apply part (ii) of Theorem 3.2 to the operator $\mathfrak{A}$. The proof is divided into eight steps.

Step 1: First, we prove that

The operator $\overline{L_{0} H_{\alpha}}$ is the generator of some Feller semigroup on $\partial \Omega$,

for any sufficiently large $\alpha>0$.

To do this, we apply Theorem 6.9 with $L:=L_{0}$.

By applying Theorem 5.1, we obtain that the oblique derivative problem

$$
\begin{cases}(A-\alpha) u=0 & \text { in } \Omega, \\ L_{0} u=\varphi & \text { on } \partial \Omega\end{cases}
$$

has a unique function $u \in W^{2, p}(\Omega)$ for any function $\varphi \in B^{1-1 / p, p}(\partial \Omega)$, if $N<p<\infty$. Here it should be noticed that we have, by an imbedding theorem for Besov spaces (see [26, Theorem 7.34]),

$$
C^{1}(\partial \Omega) \subset B^{1-1 / p, p}(\partial \Omega) \subset C(\partial \Omega)
$$

since $(1-1 / p) p=p-1>N-1$ for $N<p<\infty$.

Therefore, it follows that, for any function $\varphi \in B^{1-1 / p, p}(\partial \Omega)$ there exists a unique function $\psi \in B^{2-1 / p, p}(\partial \Omega)$ such that $L_{0}\left(H_{\alpha} \varphi\right)=\psi$. This implies that the range $R\left(L_{0} H_{\alpha}\right)$ is a dense subset of $C(\partial \Omega)$. Hence, by applying part (ii) of Theorem 6.9 with $\lambda:=0$ we obtain that the operator $\overline{L_{0} H_{\alpha}}$ generates a Feller semigroup on $\partial \Omega$, for any $\alpha>0$.

Step 2: Next we prove that

The operator $\overline{L H_{\alpha}}$ generates a Feller semigroup on $\partial \Omega$, for any $\alpha>0$.

To do this, we apply Corollary 3.3 with $K:=\partial \Omega$ to the operator $\overline{L H_{\alpha}}, \alpha>0$.

By formula (6.13), it follows that the operator $\overline{L H_{\alpha}}$ can be written in the form

$$
\begin{gathered}
\overline{L H_{\alpha}}=\overline{L_{0} H_{\alpha}}-\alpha \delta\left(x^{\prime}\right) \\
:=\overline{L_{0} H_{\alpha}}+M,
\end{gathered}
$$

where

$$
M:=-\alpha \delta\left(x^{\prime}\right)
$$

is a bounded linear operator on $C(\partial \Omega)$ into itself. However, we find that the operator $M$ satisfies condition $\left(\beta^{\prime}\right)$ of Theorem 3.2: 
$\left(\beta^{\prime}\right)$ If $\psi \in C(\partial \Omega)$ takes a positive maximum at a point $x_{0}^{\prime}$ of $\partial \Omega$, then we have

$$
M \psi\left(x_{0}^{\prime}\right)=-\alpha \delta\left(x_{0}^{\prime}\right) \psi\left(x_{0}^{\prime}\right) \leq 0 .
$$

Therefore, it follows from an application of Corollary 3.3 with $\mathfrak{A}:=\overline{L_{0} H_{\alpha}}$ $M:=-\alpha \delta\left(x^{\prime}\right)$ that the operator $\overline{L H_{\alpha}}$ also generates a Feller semigroup on $\partial \Omega$.

Step 3: Now we prove that

The equation

$$
\overline{L H_{\alpha}} \psi=\varphi
$$

has a unique solution $\psi$ in $D\left(\overline{L H_{\alpha}}\right)$ for any $\varphi \in C(\partial \Omega)$; hence

the inverse ${\overline{L H_{\alpha}}}^{-1}$ of $\overline{L H_{\alpha}}$ can be defined on the whole space $C(\partial \Omega)$.

Furthermore, the operator $-{\overline{L H_{\alpha}}}^{-1}$ is non-negative and bounded on $C(\partial \Omega)$.

Since the function $H_{\alpha} 1$ takes its positive maximum 1 only on the boundary $\partial \Omega$, we can apply the Hopf boundary point lemma (Lemma A.2) to obtain that

$$
\frac{\partial}{\partial \mathbf{n}}\left(H_{\alpha} 1\right)<0 \quad \text { on } \partial \Omega
$$

However, it should be noticed that the coefficients of the boundary condition $L$ satisfy the conditions

$$
\begin{array}{ll}
\mu\left(x^{\prime}\right)>0 & \text { on } \partial \Omega, \\
\gamma\left(x^{\prime}\right)<0 & \text { on } \partial \Omega, \\
\delta\left(x^{\prime}\right) \geq 0 & \text { on } \partial \Omega .
\end{array}
$$

Hence it follows from inequality (7.2) that

$$
L H_{\alpha} 1\left(x^{\prime}\right)=\mu\left(x^{\prime}\right) \frac{\partial}{\partial \mathbf{n}}\left(H_{\alpha} 1\right)\left(x^{\prime}\right)+\gamma\left(x^{\prime}\right)-\alpha \delta\left(x^{\prime}\right)<0 \quad \text { on } \partial \Omega,
$$

so that

$$
\ell_{\alpha}=-\sup _{x^{\prime} \in \partial \Omega} L H_{\alpha} 1\left(x^{\prime}\right)>0 .
$$

Furthermore, by using Corollary 3.3 with

$$
K:=\partial \Omega, \quad \mathfrak{A}:=\overline{L H_{\alpha}}, \quad M:=\ell_{\alpha},
$$

we obtain that the operator $\overline{L H_{\alpha}}+\ell_{\alpha} I$ is the infinitesimal generator of some Feller semigroup on $\partial \Omega$. Therefore, since $\ell_{\alpha}>0$, it follows from an application of part (i) of Theorem 3.1 with $\mathfrak{A}:=\overline{L H_{\alpha}}+\ell_{\alpha} I$ that the equation

$$
-\overline{L H_{\alpha}} \psi=\left(\ell_{\alpha} I-\left(\overline{L H_{\alpha}}+\ell_{\alpha} I\right)\right) \psi=\varphi
$$

has a unique solution $\psi \in D\left(\overline{L H_{\alpha}}\right)$ for any $\varphi \in C(\partial \Omega)$, and further that the operator $-{\overline{L H_{\alpha}}}^{-1}=\left(\ell_{\alpha} I-\left(\overline{L H_{\alpha}}+\ell_{\alpha} I\right)\right)^{-1}$ is non-negative and bounded on the space $C(\partial \Omega)$ with norm

$$
\left\|-{\overline{L H_{\alpha}}}^{-1}\right\|=\left\|\left(\ell_{\alpha} I-\left(\overline{L H_{\alpha}}+\ell_{\alpha} I\right)\right)^{-1}\right\| \leq \frac{1}{\ell_{\alpha}} .
$$


Step 4: By assertion (7.1), we can define the Green operator $G_{\alpha}, \alpha>0$, by the formula

$$
G_{\alpha} f=G_{\alpha}^{0} f-H_{\alpha}\left({\overline{L H_{\alpha}}}^{-1}\left(\overline{L G_{\alpha}^{0}} f\right)\right), \quad f \in C(\bar{\Omega}) .
$$

We prove that

$$
G_{\alpha}=(\alpha I-\mathfrak{A})^{-1}, \quad \alpha>0,
$$

where $\mathfrak{A}$ is a linear operator from $C(\bar{\Omega})$ into itself defined as follows (see formula (1.3)):

(a) The domain $D(\mathfrak{A})$ is the set

$$
D(\mathfrak{A})=\left\{u \in W^{2, p}(\Omega): u \in D(\overline{\mathcal{A}}),\left.u\right|_{\partial \Omega} \in \mathcal{D}, L u=0 \text { on } \partial \Omega\right\} .
$$

(b) $\mathfrak{A} u=A u, u \in D(\mathfrak{A})$.

Here $\mathcal{D}$ is the common domain of the operators $\overline{L H_{\alpha}}, \alpha>0$ (see Lemma 6.8).

In view of Lemmas 6.3 and 6.8 , it follows that we have, for any $f \in C(\bar{\Omega})$,

$$
\left\{\begin{array}{l}
G_{\alpha} f=G_{\alpha}^{0} f-H_{\alpha}\left({\overline{L H_{\alpha}}}^{-1}\left(\overline{L G_{\alpha}^{0}} f\right)\right) \in D(\overline{\mathcal{A}}), \\
\left.G_{\alpha} f\right|_{\partial \Omega}=-{\overline{L H_{\alpha}}}^{-1}\left(\overline{L G_{\alpha}^{0}} f\right) \in D\left(\overline{L H_{\alpha}}\right)=\mathcal{D} \\
\left.L G_{\alpha} f={\overline{L G_{\alpha}^{0}}}^{-1}-{\overline{L H_{\alpha}}}_{\overline{L H_{\alpha}}}^{-1}\left(\overline{L G_{\alpha}^{0}} f\right)\right)=0,
\end{array}\right.
$$

and also

$$
(\alpha I-\overline{\mathcal{A}}) G_{\alpha} f=f
$$

This proves that

$$
\left\{\begin{array}{l}
G_{\alpha} f \in D(\mathfrak{A}), \\
(\alpha I-\mathfrak{A}) G_{\alpha} f=f,
\end{array}\right.
$$

or equivalently,

$$
(\alpha I-\mathfrak{A}) G_{\alpha}=I \quad \text { on } C(\bar{\Omega}) .
$$

Therefore, in order to prove formula (7.2) it suffices to show the injectivity of the operator $\alpha I-\mathfrak{A}$ for $\alpha>0$.

Assume that

$$
u \in D(\mathfrak{A}) \quad \text { and } \quad(\alpha I-\mathfrak{A}) u=0 .
$$

Then, by Corollary 6.4 it follows that the function $u$ can be written as follows:

$$
u=H_{\alpha}\left(\left.u\right|_{\partial \Omega}\right),\left.\quad u\right|_{\partial \Omega} \in \mathcal{D}=D\left(\overline{L H_{\alpha}}\right) .
$$

Thus we have the assertion

$$
\overline{L H_{\alpha}}\left(\left.u\right|_{\partial \Omega}\right)=L u=0 .
$$

In view of assertion (7.1), this implies that

$$
\left.u\right|_{\partial \Omega}=0,
$$

so that

$$
u=H_{\alpha}\left(\left.u\right|_{\partial \Omega}\right)=0 \quad \text { in } \Omega .
$$


Step 5: The non-negativity of $G_{\alpha}(\alpha>0)$ follows immediately from formula (6.21), since the operators $G_{\alpha}^{0}, H_{\alpha},{\overline{L H_{\alpha}}}^{-1}$ and $\overline{L G_{\alpha}^{0}}$ are all non-negative.

Step 6: We prove that the operator $G_{\alpha}$ is bounded on the space $C(\bar{\Omega})$ with norm

$$
\left\|G_{\alpha}\right\| \leq \frac{1}{\alpha}, \quad \alpha>0
$$

To do this, it suffices to show that

$$
G_{\alpha} 1 \leq \frac{1}{\alpha} \quad \text { on } \bar{\Omega}
$$

since $G_{\alpha}$ is non-negative on $C(\bar{\Omega})$.

First, it follows from the uniqueness property of solutions of problem (6.1) (Theorem 4.1) that

$$
\alpha G_{\alpha}^{0} 1+H_{\alpha} 1=1+G_{\alpha}^{0} c \text { on } \bar{\Omega} .
$$

Indeed, it suffices to note that the both hand sides of formula (7.8) have the same boundary value 1 and satisfy the same equation: $(\alpha-A) u=\alpha$ in $\Omega$.

Applying the operator $L$ to the both hand sides of formula (7.8), we obtain that

$$
\begin{aligned}
-L H_{\alpha} 1= & -L 1-L G_{\alpha}^{0} c+\alpha L G_{\alpha}^{0} 1 \\
= & -\left(\gamma\left(x^{\prime}\right)-\delta\left(x^{\prime}\right) c\left(x^{\prime}\right)\right)-\left(\mu\left(x^{\prime}\right) \frac{\partial}{\partial \mathbf{n}}\left(G_{\alpha}^{0} c\right)+\delta\left(x^{\prime}\right) c\left(x^{\prime}\right)\right) \\
& +\alpha L G_{\alpha}^{0} 1 \\
= & -\gamma\left(x^{\prime}\right)-\mu\left(x^{\prime}\right) \frac{\partial}{\partial \mathbf{n}}\left(G_{\alpha}^{0} c\right)+\alpha L G_{\alpha}^{0} 1 \\
\geq & \alpha L G_{\alpha}^{0} 1 \quad \text { on } \partial \Omega,
\end{aligned}
$$

since $\left.G_{\alpha}^{0} c\right|_{\partial \Omega}=0$ and $G_{\alpha}^{0} c \leq 0$ on $\bar{\Omega}$. Hence we have, by the non-negativity of $-{\overline{L H_{\alpha}}}^{-1}$,

$$
-{\overline{L H_{\alpha}}}^{-1}\left(L G_{\alpha}^{0} 1\right) \leq \frac{1}{\alpha} \quad \text { on } \partial \Omega .
$$

Using formula (7.3) with $f:=1$, inequality (7.9) and formula (7.8), we obtain that

$$
\begin{aligned}
G_{\alpha} 1 & =G_{\alpha}^{0} 1+H_{\alpha}\left(-{\overline{L H_{\alpha}}}^{-1}\left(L\left(G_{\alpha}^{0} 1\right)\right)\right) \\
& \leq G_{\alpha}^{0} 1+\frac{1}{\alpha} H_{\alpha} 1 \\
& =\frac{1}{\alpha}+\frac{1}{\alpha} G_{\alpha}^{0} c \\
& \leq \frac{1}{\alpha} \text { on } \bar{\Omega}
\end{aligned}
$$

since the operators $H_{\alpha}$ and $G_{\alpha}^{0}$ are non-negative.

Step 7: Finally, we prove that

The domain $D(\mathfrak{A})$ is dense in the space $C(\bar{\Omega})$.

Step 7-1: Before the proof, we need some lemmas on the behavior of $G_{\alpha}^{0}$, $H_{\alpha}$ and $-{\overline{L H_{\alpha}}}^{-1}$ as $\alpha \rightarrow+\infty$ : 
Lemma 7.1. For all $f \in C(\bar{\Omega})$, we have the assertion

$$
\lim _{\alpha \rightarrow+\infty}\left[\alpha G_{\alpha}^{0} f+H_{\alpha}\left(\left.f\right|_{\partial \Omega}\right)\right]=f \quad \text { in } C(\bar{\Omega}) .
$$

Proof. Choose a constant $\beta>0$ and let

$$
g=f-H_{\beta}\left(\left.f\right|_{\partial \Omega}\right) .
$$

Then, by using formula (6.8) with $\varphi:=\left.f\right|_{\partial \Omega}$ we obtain that

$$
\alpha G_{\alpha}^{0} g-g=\left[\alpha G_{\alpha}^{0} f+H_{\alpha}\left(\left.f\right|_{\partial \Omega}\right)-f\right]-\beta G_{\alpha}^{0} H_{\beta}\left(\left.f\right|_{\partial \Omega}\right) .
$$

However, we have, by estimate (6.4),

$$
\lim _{\alpha \rightarrow+\infty} G_{\alpha}^{0} H_{\beta}\left(\left.f\right|_{\partial \Omega}\right)=0 \quad \text { in } C(\bar{\Omega})
$$

and, by assertion (6.7),

$$
\lim _{\alpha \rightarrow+\infty} \alpha G_{\alpha}^{0} g=g \quad \text { in } C(\bar{\Omega}),
$$

since $\left.g\right|_{\partial \Omega}=0$. Therefore, the desired assertion (7.11) follows by letting $\alpha \rightarrow$ $+\infty$ in formula (7.12).

The proof of Lemma 7.1 is complete.

Lemma 7.2. The function

$$
\frac{\partial}{\partial \mathbf{n}}\left(H_{\alpha} 1\right)\left(x^{\prime}\right), \quad x^{\prime} \in \partial \Omega,
$$

diverges to $-\infty$ uniformly and monotonically as $\alpha \rightarrow+\infty$.

Proof. First, formula (6.8) with $\varphi:=1$ gives that

$$
H_{\alpha} 1=H_{\beta} 1-(\alpha-\beta) G_{\alpha}^{0} H_{\beta} 1 .
$$

Thus, in view of the non-negativity of $G_{\alpha}^{0}$ and $H_{\alpha}$ it follows that

$$
\alpha \geq \beta \quad \Longrightarrow \quad H_{\alpha} 1 \leq H_{\beta} 1 \quad \text { on } \bar{\Omega} .
$$

Since $\left.H_{\alpha} 1\right|_{\partial \Omega}=\left.H_{\beta} 1\right|_{\partial \Omega}=1$, this implies that the functions

$$
\frac{\partial}{\partial \mathbf{n}}\left(H_{\alpha} 1\right)\left(x^{\prime}\right), \quad x^{\prime} \in \partial \Omega,
$$

are monotonically non-increasing in $\alpha$. Furthermore, by using formula (6.6) with $f:=H_{\beta} 1$ we find that the function

$$
H_{\alpha} 1(x)=H_{\beta} 1(x)-\left(1-\frac{\beta}{\alpha}\right) \alpha G_{\alpha}^{0} H_{\beta} 1(x)
$$

converges to zero monotonically as $\alpha \rightarrow+\infty$, for each interior point $x$ of $\Omega$.

Now, for any given constant $K>0$ we can construct a function $u \in W^{2, p}(\Omega)$ such that

$$
u=1 \quad \text { on } \partial \Omega
$$




$$
\frac{\partial u}{\partial \mathbf{n}} \leq-K \quad \text { on } \partial \Omega
$$

Indeed, by applying Theorem 4.1 to our situation we obtain that, for any integer $m>0$ the function

$$
u=\left(H_{1} 1\right)^{m}
$$

belongs to $W^{2, p}(\Omega)$ for $N<p<\infty$ and satisfies condition (7.13a), since we have

$$
u=\left(H_{1} 1\right)^{m}=1 \quad \text { on } \partial \Omega,
$$

and since we have

$$
\begin{aligned}
& u=\left(H_{1} 1\right)^{m} \in L^{p}(\Omega), \\
& \frac{\partial u}{\partial x_{i}}=m\left(H_{1} 1\right)^{m-1}\left(H_{1} 1\right)_{x_{i}} \in L^{p}(\Omega), \quad 1 \leq i \leq N, \\
& \frac{\partial^{2} u}{\partial x_{i} \partial x_{j}}=m\left(H_{\alpha} 1\right)^{m-1}\left(H_{1} 1\right)_{x_{i} x_{j}} \\
& \quad+m(m-1)\left(H_{\alpha} 1\right)^{m-2}\left(H_{1} 1\right)_{x_{i}}\left(H_{1} 1\right)_{x_{j}} \in L^{p}(\Omega), \quad 1 \leq i, j \leq N .
\end{aligned}
$$

Moreover, we obtain that

$$
\begin{aligned}
\frac{\partial u}{\partial \mathbf{n}} & =m \frac{\partial}{\partial \mathbf{n}}\left(H_{1} 1\right) \\
& \leq m \sup _{x^{\prime} \in \partial \Omega} \frac{\partial}{\partial \mathbf{n}}\left(H_{1} 1\right)\left(x^{\prime}\right) .
\end{aligned}
$$

In view of inequality (6.16) with $\varphi:=1$, this implies that the function $u=$ $\left(H_{1} 1\right)^{m}$ satisfies condition (7.13b) for $m$ sufficiently large.

Finally, it is easy to verify that

$$
A u \in L^{\infty}(\Omega) .
$$

Indeed, since $(A-1) H_{1} 1=0$ in $\Omega$ and $H_{1} 1 \in C^{1}(\bar{\Omega})$, it suffices to note that

$$
\begin{aligned}
A u= & m\left(H_{1} 1\right)^{m-1} \sum_{i, j=1}^{N} a^{i j}(x)\left(H_{1} 1\right)_{x_{i} x_{j}} \\
& +m(m-1)\left(H_{1} 1\right)^{m-2} \sum_{i, j=1}^{N} a^{i j}(x)\left(H_{1} 1\right)_{x_{i}}\left(H_{1} 1\right)_{x_{j}} \\
& +m\left(H_{1} 1\right)^{m-1} \sum_{i=1}^{N} b^{i}(x)\left(H_{1} 1\right)_{x_{i}}+c(x)\left(H_{1} 1\right)^{m} \\
= & m\left(H_{1} 1\right)^{m-1}\left(-\left(H_{1} 1\right)-c(x)\left(H_{1} 1\right)\right) \\
& +m(m-1)\left(H_{1} 1\right)^{m-2} \sum_{i, j=1}^{N} a^{i j}(x)\left(H_{1} 1\right)_{x_{i}}\left(H_{1} 1\right)_{x_{j}} \\
= & -m\left(H_{1} 1\right)^{m}-m c(x)\left(H_{1} 1\right)^{m}+c(x)\left(H_{1} 1\right)^{m}
\end{aligned}
$$




$$
+m(m-1)\left(H_{1} 1\right)^{m-2} \sum_{i, j=1}^{N} a^{i j}(x)\left(H_{1} 1\right)_{x_{i}}\left(H_{1} 1\right)_{x_{j}} \in L^{\infty}(\Omega) .
$$

Take a function $u$ in $W^{2, p}(\Omega)$ satisfying conditions (7.13a), (7.13b) and (7.13c), and choose a neighborhood $U$ of $\partial \Omega$, relative to $\bar{\Omega}$, with smooth boundary $\partial U$ such that

$$
u \geq \frac{1}{2} \text { on } U
$$

Recall that the function $H_{\alpha} 1$ converges to zero in $\Omega$ monotonically as $\alpha \rightarrow+\infty$. Since we have $\left.u\right|_{\partial \Omega}=\left.H_{\alpha} 1\right|_{\partial \Omega}=1$, by using Dini's theorem we can find a constant $\alpha>1$ (depending on $u$ and hence on $K$ ) such that

$$
\begin{aligned}
& H_{\alpha} 1 \leq u \quad \text { on } \partial U \backslash \partial \Omega, \\
& \alpha>2\|A u\|_{L^{\infty}(\Omega)} .
\end{aligned}
$$

It follows from inequalities $(7.14)$ and $(7.15 \mathrm{~b})$ that

$$
\begin{aligned}
(A-\alpha)\left(H_{\alpha} 1-u\right) & =\alpha u-A u \\
& \geq \frac{\alpha}{2}-\|A u\|_{L^{\infty}(\Omega)} \\
& >0 \quad \text { in } U
\end{aligned}
$$

Thus, by applying the weak maximum principle (Theorem 4.4) with $A:=A-\alpha$ to the function $H_{\alpha} 1-u$ we obtain that the function $H_{\alpha} 1-u$ may take its positive maximum only on the boundary $\partial U$. However, conditions (7.13a) and (7.15a) imply that

$$
H_{\alpha} 1-u \leq 0 \quad \text { on } \partial U=(\partial U \backslash \partial \Omega) \cup \partial \Omega .
$$

Therefore, we have

$$
H_{\alpha} 1 \leq u \quad \text { on } \bar{U}=U \cup \partial U
$$

and hence

$$
\frac{\partial}{\partial \mathbf{n}}\left(H_{\alpha} 1\right) \leq \frac{\partial u}{\partial \mathbf{n}} \leq-K \quad \text { on } \partial \Omega
$$

since $\left.u\right|_{\partial \Omega}=\left.H_{\alpha} 1\right|_{\partial \Omega}=1$ on $\partial \Omega$.

The proof of Lemma 7.2 is complete.

Corollary 7.3. $\lim _{\alpha \rightarrow+\infty}\left\|-{\overline{L H_{\alpha}}}^{-1}\right\|=0$.

Proof. Since $\mu\left(x^{\prime}\right)>0$ on $\partial \Omega$, by Lemma 7.2 it follows that the function

$$
L H_{\alpha} 1\left(x^{\prime}\right)=\mu\left(x^{\prime}\right) \frac{\partial}{\partial \mathbf{n}}\left(H_{\alpha} 1\right)\left(x^{\prime}\right)+\gamma\left(x^{\prime}\right)-\alpha \delta\left(x^{\prime}\right), \quad x^{\prime} \in \partial \Omega,
$$

diverges to $-\infty$ monotonically as $\alpha \rightarrow+\infty$. By Dini's theorem, this convergence is uniform in $x^{\prime} \in \partial \Omega$. Hence we obtain that the function

$$
\frac{1}{L H_{\alpha} 1\left(x^{\prime}\right)}
$$

converges to zero uniformly in $x^{\prime} \in \partial \Omega$ as $\alpha \rightarrow+\infty$. This implies that

$$
\left\|-{\overline{L H_{\alpha}}}^{-1}\right\|=\left\|-{\overline{L H_{\alpha}}}^{-1} 1\right\|_{C(\partial \Omega)}
$$




$$
\leq\left\|\frac{1}{L H_{\alpha} 1}\right\|_{C(\partial \Omega)} \longrightarrow 0 \quad \text { as } \alpha \rightarrow+\infty
$$

since we have

$$
1=\frac{-L H_{\alpha} 1\left(x^{\prime}\right)}{\left|L H_{\alpha} 1\left(x^{\prime}\right)\right|} \leq\left\|\frac{1}{L H_{\alpha} 1}\right\|_{C(\partial \Omega)}\left(-L H_{\alpha} 1\left(x^{\prime}\right)\right), \quad x^{\prime} \in \partial \Omega .
$$

The proof of Corollary 7.3 is complete.

Step 7-2: Proof of Assertion (7.10)

In view of formula (7.4) and inequality (7.6), it suffices to prove that

$$
\lim _{\alpha \rightarrow+\infty}\left\|\alpha G_{\alpha} f-f\right\|_{C(\bar{\Omega})}=0, \quad f \in C^{2}(\bar{\Omega}),
$$

since the space $C^{2}(\bar{\Omega})$ is dense in $C(\bar{\Omega})$.

First, we remark that

$$
\begin{aligned}
\left\|\alpha G_{\alpha} f-f\right\|_{C(\bar{\Omega})}= & \left\|\alpha G_{\alpha}^{0} f-\alpha H_{\alpha}\left({\overline{L H_{\alpha}}}^{-1}\left(\overline{L G_{\alpha}^{0}} f\right)\right)-f\right\|_{C(\bar{\Omega})} \\
\leq & \left\|\alpha G_{\alpha}^{0} f+H_{\alpha}\left(\left.f\right|_{\partial \Omega}\right)-f\right\|_{C(\bar{\Omega})} \\
& +\left\|-\alpha H_{\alpha}\left({\overline{L H_{\alpha}}}^{-1}\left(\overline{L G_{\alpha}^{0}} f\right)\right)-H_{\alpha}\left(\left.f\right|_{\partial \Omega}\right)\right\|_{C(\bar{\Omega})} \\
\leq & \left\|\alpha G_{\alpha}^{0} f+H_{\alpha}\left(\left.f\right|_{\partial \Omega}\right)-f\right\|_{C(\bar{\Omega})} \\
& +\left\|-\alpha{\overline{L H_{\alpha}}}^{-1}\left(\overline{L G_{\alpha}^{0}} f\right)-\left.f\right|_{\partial \Omega}\right\|_{C(\partial \Omega)} .
\end{aligned}
$$

Thus, in view of assertion (7.11) it suffices to show that

$$
\lim _{\alpha \rightarrow+\infty}\left[-\alpha{\overline{L H_{\alpha}}}^{-1}\left(\overline{L G_{\alpha}^{0}} f\right)-\left.f\right|_{\partial \Omega}\right]=0 \quad \text { in } C(\partial \Omega) .
$$

Take a constant $\beta$ such that $0<\beta<\alpha$, and write

$$
f=G_{\beta}^{0} g+H_{\beta} \varphi
$$

where (cf. formula (6.14)):

$$
\left\{\begin{array}{l}
g=(\beta-A) f \in L^{\infty}(\Omega) \\
\varphi=\left.f\right|_{\partial \Omega} \in C^{2}(\partial \Omega)
\end{array}\right.
$$

Then we have

$$
L G_{\beta}^{0} g=L\left(G_{\beta}^{0}(\beta-A) f\right)=L f \in C(\partial \Omega),
$$

and, by Lemma 6.6 and Remark 6.3,

$$
\begin{aligned}
L G_{\alpha}^{0} g & =L G_{\beta}^{0} g-(\alpha-\beta) \overline{L G_{\alpha}^{0}}\left(G_{\beta}^{0} g\right) \\
& =L f-(\alpha-\beta) \overline{L G_{\alpha}^{0}}(L f) \in C(\partial \Omega) .
\end{aligned}
$$

Moreover, by using the resolvent equation (6.5) with $f:=g \in L^{\infty}(\Omega)$ and the equation (6.8), we obtain that

$$
G_{\alpha}^{0} f=G_{\alpha}^{0}\left(G_{\beta}^{0} g\right)+G_{\alpha}^{0} H_{\beta} \varphi
$$




$$
=\frac{1}{\alpha-\beta}\left(G_{\beta}^{0} g-G_{\alpha}^{0} g+H_{\beta} \varphi-H_{\alpha} \varphi\right) .
$$

Therefore, it follows that

$$
\begin{aligned}
& \left\|-\alpha{\overline{L H_{\alpha}}}^{-1}\left(\overline{L G_{\alpha}^{0}} f\right)-\left.f\right|_{\partial \Omega}\right\|_{C(\partial \Omega)} \\
= & \left\|\frac{\alpha}{\alpha-\beta}\left(-{\overline{L H_{\alpha}}}^{-1}\right)\left(L G_{\beta}^{0} g-L G_{\alpha}^{0} g+L H_{\beta} \varphi\right)+\frac{\alpha}{\alpha-\beta} \varphi-\varphi\right\|_{C(\partial \Omega)} \\
= & \left\|\frac{\alpha}{\alpha-\beta}\left(-{\overline{L H_{\alpha}}}^{-1}\right)\left(\left(L f+L H_{\beta} \varphi\right)-L G_{\alpha}^{0} g\right)+\frac{\beta}{\alpha-\beta} \varphi\right\|_{C(\partial \Omega)} \\
\leq & \frac{\alpha}{\alpha-\beta}\left\|-{\overline{L H_{\alpha}}}^{-1}\right\| \cdot\left\|L f+L H_{\beta} \varphi\right\|_{C(\partial \Omega)} \\
& +\frac{\alpha}{\alpha-\beta}\left\|-{\overline{L H_{\alpha}}}^{-1}\right\| \cdot\left\|L G_{\alpha}^{0}\right\| \cdot\|g\|_{L^{\infty}(\Omega)}+\frac{\beta}{\alpha-\beta}\|\varphi\|_{C(\partial \Omega)} .
\end{aligned}
$$

By Corollary 7.3, it follows that the first term on the last inequality (7.18) converges to zero as $\alpha \rightarrow+\infty$. For the second term, by using the resolvent equation (6.5) with $f:=1$ and the non-negativity of $G_{\beta}^{0}$ and $L G_{\alpha}^{0}$ (Remark 6.2) we find that

$$
\begin{aligned}
\left\|L G_{\alpha}^{0}\right\| & =\left\|L\left(G_{\alpha}^{0} 1\right)\right\|_{C(\partial \Omega)} \\
& =\left\|L\left(G_{\beta}^{0} 1\right)-(\alpha-\beta) \overline{L G_{\alpha}^{0}}\left(G_{\beta}^{0} 1\right)\right\|_{C(\partial \Omega)} \\
& \leq\left\|L\left(G_{\beta}^{0} 1\right)\right\|_{C(\partial \Omega)} .
\end{aligned}
$$

Hence the second term on the last inequality (7.18) also converges to zero as $\alpha \rightarrow+\infty$. It is clear that the third term on the last inequality (7.18) converges to zero as $\alpha \rightarrow+\infty$. This completes the proof of assertion (7.17) and hence of assertion (7.16).

Step 8: Summing up, we have proved that the operator $\mathfrak{A}$, defined by formula (7.5), satisfies conditions (a) through (d) in Theorem 3.2. Hence it follows from an application of the same theorem that the operator $\mathfrak{A}$ is the infinitesimal generator of some Feller semigroup on $\bar{\Omega}$.

The proof of Theorem 1.1 is now complete. 


\section{Concluding Remarks}

For more general results on generation theorems for Feller semigroups, we give the following overview:

\begin{tabular}{|c|c|c|c|c|}
\hline $\begin{array}{c}\text { diffusion } \\
\text { operator } \\
A\end{array}$ & $\begin{array}{c}\text { Lévy } \\
\text { operator } \\
S\end{array}$ & $\begin{array}{c}\text { Ventcel' } \\
\text { condition } \\
L\end{array}$ & $\begin{array}{c}\text { using } \\
\text { the theory } \\
\text { of }\end{array}$ & $\begin{array}{l}\text { proved } \\
\text { by }\end{array}$ \\
\hline $\begin{array}{l}\text { smooth } \\
\text { coefficients } \\
\text { case }\end{array}$ & null & $\begin{array}{l}\text { second-order } \\
\text { case }\end{array}$ & $\begin{array}{l}\text { pseudo- } \\
\text { differential } \\
\text { operators }\end{array}$ & {$[13]$} \\
\hline $\begin{array}{l}\text { smooth } \\
\text { coefficients } \\
\text { case }\end{array}$ & $\begin{array}{l}\text { general } \\
\text { case }\end{array}$ & $\begin{array}{l}\text { general } \\
\text { case }\end{array}$ & $\begin{array}{l}\text { pseudo- } \\
\text { differential } \\
\text { operators }\end{array}$ & [33] \\
\hline $\begin{array}{l}\text { smooth } \\
\text { coefficients } \\
\text { case }\end{array}$ & $\begin{array}{c}\text { Hölder } \\
\text { continuous } \\
\text { case }\end{array}$ & $\begin{array}{c}\text { degenerate } \\
\text { Robin } \\
\text { case } L_{1}\end{array}$ & $\begin{array}{c}\text { pseudo- } \\
\text { differential } \\
\text { operators }\end{array}$ & {$[6]$} \\
\hline $\begin{array}{c}\text { VMO } \\
\text { coefficients } \\
\text { case }\end{array}$ & $\begin{array}{l}\text { general } \\
\text { case }\end{array}$ & $\begin{array}{l}\text { Dirichlet } \\
\text { case }\end{array}$ & $\begin{array}{c}\text { singular } \\
\text { integral } \\
\text { operators }\end{array}$ & {$[15]$} \\
\hline $\begin{array}{c}\text { VMO } \\
\text { coefficients } \\
\text { case }\end{array}$ & null & $\begin{array}{l}\text { first-order } \\
\text { case }\end{array}$ & $\begin{array}{c}\text { singular } \\
\text { integral } \\
\text { operators }\end{array}$ & $\begin{array}{l}\text { the } \\
\text { present } \\
\text { paper }\end{array}$ \\
\hline
\end{tabular}

Here the boundary condition $L_{1}$ of Robin type is given by the formula

$$
L_{1} u:=\mu\left(x^{\prime}\right) \frac{\partial u}{\partial \mathbf{n}}+\gamma\left(x^{\prime}\right) u \quad \text { on } \partial \Omega,
$$

where the coefficients $\mu\left(x^{\prime}\right)$ and $\gamma\left(x^{\prime}\right)$ satisfy the following three conditions (i), (ii) and (iii):

(i) $\mu\left(x^{\prime}\right)$ is a smooth function on $\partial \Omega$ and $\mu\left(x^{\prime}\right) \geq 0$ on $\partial \Omega$.

(ii) $\gamma\left(x^{\prime}\right)$ is a smooth function on $\partial \Omega$ and $\gamma\left(x^{\prime}\right) \leq 0$ on $\partial \Omega$.

(iii) $\mu\left(x^{\prime}\right)+\left|\gamma\left(x^{\prime}\right)\right|>0$ on $\partial \Omega$.

It should be emphasized that $L_{1}$ becomes a degenerate boundary condition from an analytical point of view. This is due to the fact that the so-called ShapiroLopatinskii complementary condition is violated at the points $x^{\prime} \in \partial \Omega$ where $\mu\left(x^{\prime}\right)=0$. 


\section{A The Maximum Principle}

In this appendix we formulate various maximum principles for second-order elliptic differential operators with discontinuous coefficients such as the weak and strong maximum principles (Theorems A.1 and A.3) and the Hopf boundary point lemma (Lemma A.2) in the framework of Sobolev spaces of $L^{p}$. The results here are adapted from Bony [16] and also Taira [13].

Let $\Omega$ be a bounded domain in Euclidean space $\mathbf{R}^{N}, N \geq 2$, with boundary $\partial \Omega$ of class $C^{1,1}$. We consider a second-order elliptic differential operator $A$ with real discontinuous coefficients of the form

$$
A u:=\sum_{i, j=1}^{N} a^{i j}(x) \frac{\partial^{2} u}{\partial x_{i} \partial x_{j}}+\sum_{i=1}^{N} b^{i}(x) \frac{\partial u}{\partial x_{i}}+c(x) u .
$$

More precisely, we assume that the coefficients $a^{i j}(x), b^{i}(x)$ and $c(x)$ of the differential operator $A$ satisfy the following three conditions (1), (2) and (3):

(1) $a^{i j}(x) \in L^{\infty}(\Omega), a^{i j}(x)=a^{j i}(x)$ for almost all $x \in \Omega$ and there exist a constant $\lambda>0$ such that

$$
\frac{1}{\lambda}|\xi|^{2} \leq \sum_{i, j=1}^{N} a^{i j}(x) \xi_{i} \xi_{j} \leq \lambda|\xi|^{2} \quad \text { for almost all } x \in \Omega \text { and all } \xi \in \mathbf{R}^{N} .
$$

(2) $b^{i}(x) \in L^{\infty}(\Omega)$.

(3) $c(x) \in L^{\infty}(\Omega)$ and $c(x) \leq 0$ for almost all $x \in \Omega$.

First, we state a variant of the weak maximum principle in the framework of Sobolev spaces of $L^{p}$ style, due to Bony [16]:

Theorem A.1. If a function $u \in W^{2, p}(\Omega), N<p<\infty$, satisfies the condition

$$
A u(x) \geq 0 \quad \text { for almost all } x \in \Omega,
$$

then we have the inequality

$$
\max _{\bar{\Omega}} u \leq \max _{\partial \Omega} u^{+}
$$

where

$$
u^{+}(x)=\max \{u(x), 0\}, \quad x \in \bar{\Omega} .
$$

Secondly, the Hopf boundary point lemma reads as follows:

Lemma A.2 (Hopf). Assume that a function $u \in W^{2, p}(\Omega), N<p<\infty$, satisfies the condition

$$
A u(x) \geq 0 \quad \text { for almost all } x \in \Omega \text {. }
$$

If $u(x)$ attains a non-negative, strict local maximum at a point $x_{0}^{\prime}$ of $\partial \Omega$, then we have the inequality

$$
\frac{\partial u}{\partial \mathbf{n}}\left(x_{0}^{\prime}\right)<0
$$


Finally, we can obtain the following strong maximum principle for the operator $A$ ( [16, Théorème 2]):

Theorem A.3. Assume that a function $u \in W^{2, p}(\Omega), N<p<\infty$, satisfies the condition

$$
A u(x) \geq 0 \quad \text { for almost all } x \in \Omega .
$$

If $u(x)$ attains a non-negative maximum at an interior point $x_{0}$ of $\Omega$, then it is a (non-negative) constant function.

\section{References}

[1] Gilbarg, D. and Trudinger, N.S., Elliptic partial differential equations of second order, 1998 edition, Springer-Verlag New York Berlin Heidelberg Tokyo, 1998.

[2] Meyers, N., An $L^{p}$-estimate for the gradient of solutions of second order elliptic divergence equations, Ann. Scuola Norm. Sup. Pisa 17, 189-206 (1963).

[3] Miranda, C., Sulle equazioni ellittiche del secondo ordine di tipo non variazionale a coefficienti discontinui, Ann. Mat. Pura Appl. 63, 353-386 (1963).

[4] Talenti, G., Equazioni lineari ellittiche in due variabili, Matematiche (Catania) 21, 339-376 (1966).

[5] Calderón, A.P. and Zygmund, A., On the existence of certain singular integrals, Acta. Math. 88, 85-139 (1952).

[6] Taira, K., Semigroups, boundary value problems and Markov processes, Springer-Verlag, Berlin Heidelberg New York, 2004.

[7] Chiarenza, F., Frasca, M. and Longo, P., Interior $W^{2, p}$ estimates for nondivergence elliptic equations with discontinuous coefficients, Ricerche di Matematica 60, 149-168 (1991).

[8] Chiarenza, F., Frasca, M. and Longo, P., $W^{2, p}$-solvability of the Dirichlet problem for nondivergence elliptic equations with VMO coefficients, Trans. Amer. Math. Soc. 336, 841-853 (1993).

[9] Bramanti, M., Commutators of integral operators with positive kernels, Matematiche (Catania) 49, 149-168 (1994).

[10] Stein, E.M., Harmonic analysis: real-variable methods, orthogonality, and oscillatory integrals, Princeton Univ. Press, Princeton, 1993.

[11] Wentzell (Ventcel'), A.D., On boundary conditions for multidimensional diffusion processes (in Russian), Teoriya Veroyat. $i$ ee Primen. 4, 172185 (1959); English translation in Theory Prob. and its Appl. 4, 164-177 (1959).

[12] Dynkin, E.B., Markov processes I, II, Springer-Verlag, Berlin Göttingen Heidelberg, 1965. 
[13] Taira, K., Diffusion processes and partial differential equations, Academic Press, San Diego New York London Tokyo, 1988.

[14] Dynkin, E.B., Foundations of the theory of Markov processes, Fizmatgiz, Moscow, 1959 (in Russian); English translation: Pergamon Press, Oxford London New York Paris, 1960; German translation: Springer-Verlag, Berlin Göttingen Heidelberg, 1961; French translation: Dunod, Paris, 1963.

[15] Taira, K., On the existence of Feller semigroups with discontinuous coefficients, Acta Math. Sinica (English Series) 22, 595-606 (2006).

[16] Bony, J.-M., Principe du maximum dans les espaces de Sobolev, C. R. Acad. Sc. Paris 265, 333-336 (1967).

[17] Lieberman, G.M., Local estimates for subsolutions and supersolutions of oblique derivative problems for general second order elliptic equations, Trans. Amer. Math. Soc. 304, 343-353 (1987).

[18] Garnett, J.B., Bounded analytic functions, Academic Press, New York London Toronto Sydney San Francisco, 1981.

[19] Torchinsky, A., Real-variable methods in harmonic analysis, Academic Press, Orlando San Diego New York London Tokyo, 1986.

[20] John, F. and Nirenberg, L., On functions of bounded mean oscillation, Comm. Pure and Appl. Math. 14, 415-426 (1961).

[21] Neri, U., Some properties of functions with bounded mean oscillation, Studia Math. 61, 63-75 (1977).

[22] Sarason, D., Functions of vanishing mean oscillation, Trans. Amer. Math. Soc. 207, 391-405 (1975).

[23] Acquistapace, P., On BMO regularity for linear elliptic systems, Ann. Mat. Pura Appl. 161, 231-269 (1992).

[24] Bony, J.-M., Courrège, P. and Priouret, P., Semi-groupes de Feller sur une variété à bord compacte et problèmes aux limites intégro-différentiels du second ordre donnant lieu au principe du maximum, Ann. Inst. Fourier (Grenoble) 18, 369-521 (1968).

[25] Yosida, K., Functional analysis, sixth edition, Springer-Verlag, Berlin Heidelberg New York, 1980.

[26] Adams, R.A. and Fournier, J.J.F., Sobolev spaces, second edition, Academic Press, Amsterdam Heidelberg New York Oxford, 2003.

[27] Bergh, J. and Löfström, J., Interpolation spaces, an introduction, SpringerVerlag, Berlin Heidelberg New York, 1976.

[28] Triebel, H., Theory of function spaces, Birkhäuser, Basel Boston Stuttgart, 1983.

[29] Maugeri, A. and Palagachev, D.K., Boundary value problem with an oblique derivative for uniformly elliptic operators with discontinuous coefficients, Forum Math. 10, 393-405 (1998). 
[30] Maugeri, A., Palagachev, D.K. and Softova, L.G., Elliptic and parabolic equations with discontinuous coefficients, Mathematical Research, 109, Wiley-VCH, Berlin, 2000.

[31] Amann, H., Nonlinear elliptic equations with nonlinear boundary conditions, In: New developments in differential equations, W. Eckhaus (ed.), North-Holland Mathematics Studies, vol. 21 (North-Holland, Amsterdam New York Oxford), 43-63 (1976).

[32] Sato, K. and Ueno, T., Multi-dimensional diffusion and the Markov process on the boundary, J. Math. Kyoto Univ. 14, 529-605 (1965).

[33] Taira, K., On the existence of Feller semigroups with boundary conditions III, Hiroshima Math. J. 27, 77-103 (1997). 